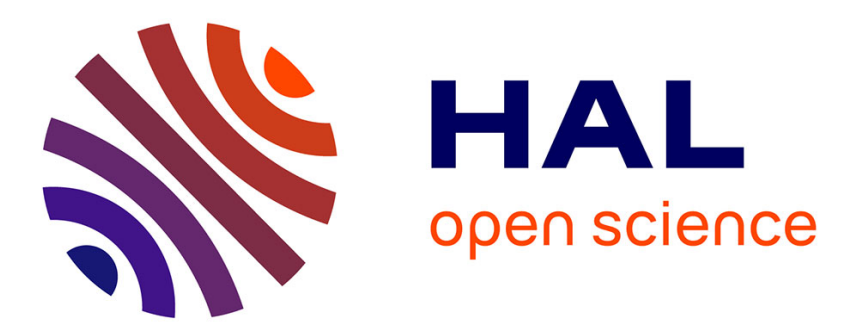

\title{
IRIS: a new generation of IRAS maps
}

\author{
Marc-Antoine Miville-Deschênes, Guilaine Lagache
}

\section{To cite this version:}

Marc-Antoine Miville-Deschênes, Guilaine Lagache. IRIS: a new generation of IRAS maps. The Astrophysical Journal Supplement, 2005, 10.1086/427938 . hal-01840563

\section{HAL Id: hal-01840563 \\ https://hal-amu.archives-ouvertes.fr/hal-01840563}

Submitted on 16 Jul 2018

HAL is a multi-disciplinary open access archive for the deposit and dissemination of scientific research documents, whether they are published or not. The documents may come from teaching and research institutions in France or abroad, or from public or private research centers.
L'archive ouverte pluridisciplinaire HAL, est destinée au dépôt et à la diffusion de documents scientifiques de niveau recherche, publiés ou non, émanant des établissements d'enseignement et de recherche français ou étrangers, des laboratoires publics ou privés. 


\title{
IRIS: A NEW GENERATION OF IRAS MAPS
}

\author{
Marc-Antoine Miville-DeschêNes \\ Canadian Institute for Theoretical Astrophysics, 60 St-George Street, Toronto, ON, M5S 3H8, Canada; mamd@cita.utoronto.ca \\ AND \\ Guilaine Lagache \\ Institut d'Astrophysique Spatiale, Université Paris-Sud, Bât. 121, 91405, Orsay, France; lagache@ias.u-psud.fr \\ Received 2004 April 27; accepted 2004 December 8
}

\begin{abstract}
The Infrared Astronomical Satellite (IRAS) had a tremendous impact on many areas of modern astrophysics. In particular, it revealed the ubiquity of infrared cirrus that are a spectacular manifestation of the interstellar medium complexity but also an important foreground for observational cosmology. With the forthcoming Planck satellite there is a need for all-sky complementary data sets with arcminute resolution that can bring informations on specific foreground emissions that contaminate the cosmic microwave background radiation. With its $\sim 4^{\prime}$ resolution matching perfectly the high-frequency bands of Planck, IRAS is a natural data set to study the variations of dust properties at all scales. But the latest version of the images delivered by the IRAS team (the ISSA plates) suffer from calibration, zero level, and striping problems that can preclude its use, especially at 12 and $25 \mu \mathrm{m}$. In this paper we present how we proceeded to solve each of these problems and enhance significantly the general quality of the ISSA plates in the four bands $(12,25,60$, and $100 \mu \mathrm{m})$. This new generation of IRAS images, called IRIS, benefits from a better zodiacal light subtraction, from a calibration and zero level compatible with DIRBE, and from a better destriping. At $100 \mu \mathrm{m}$ the IRIS product is also a significant improvement from the Schlegel et al. maps. IRIS keeps the full ISSA resolution, it includes well-calibrated point sources, and the diffuse emission calibration at scales smaller than $1^{\circ}$ was corrected for the variation of the IRAS detector responsivity with scale and brightness. The uncertainty on the IRIS calibration and zero level is dominated by the uncertainty on the DIRBE calibration and on the accuracy of the zodiacal light model.
\end{abstract}

Subject headings: astronomical data bases: miscellaneous — infrared: general — surveys

Online material: color figure

\section{INTRODUCTION}

From 1983 January to November the Infrared Astronomical Satellite (IRAS), a joint project of the US, UK, and the Netherlands (Neugebauer et al. 1984), performed a survey of $98 \%$ of the sky at four wavelengths: $12,25,60$, and $100 \mu \mathrm{m}$. IRAS led to numerous scientific discoveries spanning a broad range of astrophysical subjects, from comets to circumstellar disks to interacting galaxies. The satellite was designed to optimize the reliability of point source detection and photometry; one of the great legacies of IRAS is certainly its catalog of more than 250,000 point sources. On the other hand, the relative stability of its detectors also allowed mapping of extended emission. In fact, IRAS made a significant contribution to our understanding of Galactic diffuse emission by revealing the interstellar dust emission of infrared cirrus, which can be observed in any direction on the sky (Low et al. 1984).

A first set of extended emission maps (the SkyFlux atlas) was released in 1984 and 1986 along with the IRAS Point Source Catalog. It was then clear that significant improvements of the sensitivity and photometric accuracy could be obtained based on the acquired knowledge of the instrument. The IRAS data were later reprocessed based on the knowledge of the instrumental response available at that time but also by applying a zodiacal light correction and a destriping method. This second generation processing, which increased the sensitivity by up to a factor of 5, led to the IRAS Sky Survey Atlas (ISSA) published in 1991 and 1992. The IRAS Sky Survey Atlas Explanatory Supplement (Wheelock et al. 1993) describes in detail the processing and analysis of this atlas.
Since its publication, the ISSA has been a tremendously useful data product, used to study various aspects of Galactic (e.g., Boulanger \& Pérault 1988) and extragalactic (e.g., MivilleDeschênes et al. 2002b) diffuse emission. The ISSA plates have become an essential data set for any multiwavelength analysis of the interstellar medium. Unfortunately, even after the heroic processing done by the IRAS team, the ISSA plates still suffer from defects (striping, calibration, zero level, zodiacal light) that can limit significantly their use. In the context of the numerous ongoing and future infrared $/ \mathrm{submm} / \mathrm{mm}$ missions (Spitzer, Herschel, Planck, JWST, ASTRO-F, ...), IRAS data could still be highly valuable, even with their relatively coarse angular resolution $\left(\sim 4^{\prime}\right)$. This was our main motivation to reprocess the ISSA plates of all IRAS bands, in order to improve their sensitivity and absolute calibration.

This new generation of IRAS maps, a product named IRIS (for Improved Reprocessing of the IRAS Survey), will be of particular interest for the Planck mission dedicated to the study of the cosmic microwave background (CMB). Planck will perform an all-sky survey in the $350 \mu \mathrm{m}-10 \mathrm{~mm}$ wavelength range with an angular resolution in the high-frequency bands comparable to IRAS. One of the main anticipated challenges of this mission will be the separation of all the components, including dust, that contribute to the signal in this wavelength range (as it is for WMAP; Bennett et al. 2003). Several studies of dust emission at high Galactic latitudes showed large variations of dust properties at all scales, even in the diffuse interstellar medium (Boulanger et al. 1990; Lagache et al. 1998; Bernard et al. 1999; Miville-Deschênes et al. 2002a; Stepnik et al. 2003). To improve on the dust models available (e.g., Finkbeiner et al. 1999) 
and bring them to an accuracy level required for Planck, dust evolution should be understood in a more general way, including the properties of the smaller grain populations that give some informations on the evolutionary stage of dust (coagulation, fragmentation) and on the properties of the bigger grains that contribute to the submm $/ \mathrm{mm}$ emission. Small grains have also been suggested (Draine \& Lazarian 1998) to be responsible for the anomalous microwave emission (e.g., Lagache 2003). In this context, well-calibrated, arcminute resolution and allsky dust emission maps at 12, 25, 60, and $100 \mu \mathrm{m}$ would be of tremendous help to understand and model dust emission in the Planck bands. This is one of the main motivations of the present work.

The present paper describes in detail the procedure we followed to reduce significantly the striping of the ISSA maps and to rescale them to have an absolute calibration coherent with both the DIRBE and IRAS Point Source Catalog photometry. In $\S 2$ we describe in detail the ISSA product. Our reprocessing of the ISSA plates, which includes an automatic deglitching, an improved destriper, and an absolute calibration and zero level correction, is described in $\S \S 3$ and 4 . The overall properties of the IRIS product are discussed in $\S 5$. The whole IRIS data set is available online. ${ }^{1}$

\section{THE IRAS SKY SURVEY ATLAS}

\subsection{Processing of the ISSA Plates}

During its 10 months operation period, IRAS made two surveys of $98 \%$ of the sky and a third one of $75 \%$ of the sky. To confirm point source detection, each survey, called an HCON for Hours Confirmation, consisted of two coverages of the sky separated by up to 36 hours. The first two HCONs (HCON-1 and HCON-2) were done concurrently with the second one behind the first one by a few weeks. The third survey (HCON-3) began after the first two were completed. Owing to exhaustion of the liquid helium supply, the third HCON could not be completed and it thus covers only $75 \%$ of the sky.

The ISSA is a set of 430 fields of $500 \times 500$ pixels of 1.'5. Each field is a $12^{\circ} .5 \times 12^{\circ} .5$ region centered every $10^{\circ}$ in declination. All HCONs represent the sky surface brightness at the four wavelengths from which an estimate of the zodiacal light was subtracted. The HCONs were destripped, checked for zero level stability, and visually examined for artifacts and glitches. The processed HCONs were then co-added, using sky coverage maps, to produce one final map (named $\mathrm{HCON}-0$ ) per field and per wavelength.

\subsection{Diffuse Emission Photometry}

Special care has been taken to have a consistent diffuse emission calibration through the Atlas. But, as the IRAS mission was designed to provide absolute photometry for point sources only, the ISSA images give only relative photometry on spatial scales larger than $\sim 5^{\prime}$ and cannot be used directly to determine the absolute surface brightness of diffuse emission. The uncertainty on the zero level is dominated by uncertainties in the zodiacal light model at 12 and $25 \mu \mathrm{m}$ and by detector drifts at 60 and $100 \mu \mathrm{m}$.

As it was designed to study the cosmic infrared background, the DIRBE (Diffuse Infrared Background Experiment) experiment on board the COBE (Cosmic Background Explorer)

\footnotetext{
${ }^{1}$ See http://www.ias.fr/IRIS and http://www.cita.utoronto.ca/ mamd/IRIS
}

satellite has made absolute surface brightness measurements of the sky at scales larger than 0.7 . Moreover, the DIRBE data were corrected with a better zodiacal light model (Kelsall et al. 1998) than the one used for the ISSA. Fortunately, DIRBE and $I R A S$ had very similar filters, and therefore the DIRBE data can be used to determine the absolute $I R A S$ calibration at scales larger than $0^{\circ} 7$. Some efforts have been put in the comparison between DIRBE data and individual HCON images. Wheelock et al. (1993) showed that the amplitude of the 60 and $100 \mu \mathrm{m}$ intensity fluctuations are overestimated in the ISSA maps with respect to DIRBE. Wheelock et al. (1993) suggest a linear relation between the IRAS and DIRBE surface brightness that also takes into account the difference in zero level:

$$
I_{\lambda}^{\mathrm{DIRBE}}(\alpha, \delta)=G_{\lambda} \times I_{\lambda}^{\mathrm{ISSA}}(\alpha, \delta)+B_{\lambda}(\alpha, \delta) .
$$

The gain correction factors $\left(G_{\lambda}\right)$ given in Table 1 were originally obtained on spatial scales of the order of an IRAS scan $\left(\sim 8^{\circ}\right)$. We have performed a detailed comparison of the DIRBE and IRAS data on numerous regions covering a broad range of brightness, and we conclude that these factors vary with angular scale and brightness. This issue is discussed in detail in $\S 4.3$. Regarding the zero point of the ISSA maps, Schlegel et al. (1998) showed that the $100 \mu \mathrm{m}$ offset $B_{100}(\alpha, \delta)$ varies significantly across the sky. Our analyses show that this is the case at all wavelengths (see $\S 4.5)$.

\subsection{Point Source Photometry}

By staring at point sources, the IRAS team clearly showed (Wheelock et al. 1993) that the instantaneous (AC) and longterm (DC) responsivity of the IRAS detectors were different. For a survey mission like $I R A S$, this introduces a responsivity difference between pointlike object and diffuse emission. The ISSA plates are DC calibrated, which means that point source fluxes estimated on a ISSA plate have to be corrected for the DC/AC responsivity difference (see Table 1). Even if the ISSA product was not optimized for point sources, Wheelock et al. (1993) showed that, when corrected for the appropriate DC/AC factor, point source fluxes measured on ISSA plates are photometrically consistent with the IRAS Point Source Catalog to within $10 \%$. We have verified this by comparing the DIRBE and ISSA (convolved by the DIRBE beam) fluxes of several stars. We also found a general good agreement between these two data sets.

\subsection{Postprocessing of the ISSA Plates}

Beside the responsivity variation and zero level problems, the ISSA plates have also relatively strong stripes, related to the scanning on the sky, that participate significantly to the noise. The main goals of the present work were first to reduce the amplitude of those stripes to lower the noise level and second to correct the ISSA plates calibration to match both the DIRBE and the Point Source Catalog photometry.

In the next sections we describe how we have addressed all of these problems. First we discuss how we have deglitched automatically the individual HCONs ( $\S 3.1)$. Then we describe our destriping algorithm ( $\S 3.2$ ). Finally, we describe how we use the comparison of the IRAS and DIRBE data to establish a calibration and a zero level correction that is coherent both for point sources and diffuse emission $(\S 4)$.

Throughout the processing we have taken great care to work on individual HCONs in order to improve the destriping of the 
TABLE 1

Compilation of IRIS Related Quantities

\begin{tabular}{|c|c|c|c|c|}
\hline \multirow[b]{2}{*}{ QuANTITY } & \multicolumn{4}{|c|}{ WAVELENGTH } \\
\hline & $12 \mu \mathrm{m}$ & $25 \mu \mathrm{m}$ & $60 \mu \mathrm{m}$ & $100 \mu \mathrm{m}$ \\
\hline 1. IRAS resolution $(\operatorname{arcmin})$ & $0.75 \times 4.5$ & $0.75 \times 4.6$ & $1.5 \times 4.7$ & $3.0 \times 5.0$ \\
\hline 2. IRIS resolution $(\operatorname{arcmin}) \ldots \ldots \ldots \ldots \ldots \ldots \ldots \ldots$ & $3.8 \pm 0.2$ & $3.8 \pm 0.2$ & $4.0 \pm 0.2$ & $4.3 \pm 0.2$ \\
\hline 3. ISSA noise level $\left(\mathrm{MJy} \mathrm{sr}^{-1}\right) \ldots \ldots \ldots \ldots \ldots \ldots \ldots$ & $0.04 \pm 0.01$ & $0.05 \pm 0.02$ & $0.04 \pm 0.01$ & $0.07 \pm 0.03$ \\
\hline 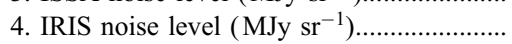 & $0.04 \pm 0.01$ & $0.05 \pm 0.02$ & $0.03 \pm 0.01$ & $0.06 \pm 0.02$ \\
\hline 5. DC/AC & 0.78 & 0.82 & 0.92 & 1.00 \\
\hline 6. $G_{\lambda} \ldots \ldots$ & $1.06 \pm 0.02$ & $1.01 \pm 0.02$ & $0.87 \pm 0.05$ & $0.72 \pm 0.07$ \\
\hline 7. DIRBE $S$ (gain) $(\%)$ & 5.1 & 15.1 & 10.4 & 13.5 \\
\hline 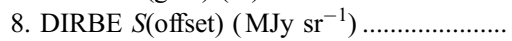 & $6.0 \times 10^{-5}$ & $8.3 \times 10^{-5}$ & 0.027 & 0.027 \\
\hline
\end{tabular}

Notes.-Row 1: Effective instrumental resolution of the IRAS detectors (Wheelock et al. 1993). Row 2: Angular resolution of the ISSA and IRIS plates (see $\S 5.2$ ). Rows 3-4: The ISSA and IRIS noise level given here are representative of the median instrumental noise of the whole plates. The noise level varies from one plate to the other as a function of coverage (see $\S 5.1$ ). Rows 5-6: DC/AC and IRAS/DIRBE corrections given by the IRAS team (Wheelock et al. 1993). Rows 7-8: DIRBE gain and offset uncertainties from Hauser et al. (1998).

maps and to be able to estimate the noise level in the final averaged maps. This specific point is discussed in $\S 5.1$.

\section{CLEANING THE ISSA PLATES}

\subsection{Deglitching}

Before co-adding the individual HCONs, the IRAS team inspected them visually to identify artifacts like moving objects (e.g., asteroids) or residual glitches. To allow for further validity checks of point sources seen (or not) in the co-added maps, the IRAS team decided to provide the individual HCONs that include those artifacts. As we are working directly on the individual $\mathrm{HCONs}$ we had to redo the deglitching but we took advantage of the fact that the co-added maps ( $\mathrm{HCON}-0)$ have already been deglitched.

First, using the method described in Appendix A we identified point sources both in the individual HCONs and in the coadded map. In a given map the identified pixels were then gathered together into regions by consecutively labeling all the detecting regions with a unique region index. ${ }^{2}$ Then we computed the central position (taken as the position of the brightest pixel) and the maximum radius of each region. For each region found in the individual $\operatorname{HCON}(1,2$, or 3$)$ we derived the distance to all regions found in the co-added map. Each HCON region with no counterpart in the co-added map at distances lower than the sum of the two regions maximum radius is considered as a glitch. The corresponding pixels in the HCON are then set to the background level at that position.

This automatic method worked very well to identify automatically residual glitches and moving objects on most of the ISSA plates. It did not perform as well in very bright regions were the diffuse emission varies significantly at small scales. Therefore, we did not deglitch regions brighter than $200 \mathrm{MJy}$ $\mathrm{sr}^{-1}$ at $100 \mu \mathrm{m}$. We also dealt manually with Saturn because of its large extension in the affected HCONs.

\subsection{Destriping}

The ISSA plates have relatively strong stripes that are related to the scanning on the sky. We describe in this part our destriping algorithm that reduces significantly the amplitude of those stripes. The destriping is done in Fourier space for each

\footnotetext{
${ }^{2}$ We use the "label_region" function of IDL.
}

HCON. For typical HCONs, the stripes have one or two directions but there exists more complicated regions where there are several directions (up to 5 for example near the Large Magellanic Cloud). In the Fourier space, such stripes give extra power in well-defined azimuthal directions that can be identified and corrected (Fig. 1).

To be able to identify clearly the striping pattern in Fourier space, two effects have to be dealt with: pointlike sources and large-scale gradients. Point sources introduce contaminating power in Fourier space in every direction and with a flat power spectrum. Their main effect is to lower significantly the contrast of stripes in Fourier space, especially at large wavenumbers $k$. On the contrary, a large-scale gradient across an image produces extra power in privileged directions in Fourier space (horizontal and vertical) that mimics stripes. To get rid of this effect it is quite common to apodize the image (see Schlegel et al. 1998 for example) but this destroys the information on the edge of the image.

To get around these difficulties we used a wavelet decomposition ("a trou" algorithm) of every HCON and worked only on an image that is the sum of the first five scales $(2,4,8,16$, and 32 pixels of 1.'5). This filtered map contains only small-scale structures, usually dominated by the stripes, and is thus free of any large-scale gradient (Fig. 1). To increase the contrast of the striping pattern we removed point sources at each scale of the wavelet decomposition prior to summing them; we clipped all the wavelet coefficients higher than $7 \sigma$, where $\sigma$ is computed at each scale. The original HCON was then decomposed into three components: a large-scale emission map (scales larger than 32 pixels), small-scale emission map (scales smaller than 32 pixels), and a point sources map. The following destriping algorithm was only applied on the small-scale emission map.

Each small-scale emission map was fast Fourier transformed (FFT), and the averaged magnitude of the FFT was computed as a function of angle $\theta$ in 360 bins of $1^{\circ}$ wide. We identified the bins with averaged magnitude greater than $N \sigma$ and replaced them by the averaged azimuthal magnitude. This is equivalent to replace the identified bins by the power spectrum of the clean bins. This procedure was repeated twice on an artificially rotated FFT pattern (to detect stripes with direction falling between two bins). During this process, we progressively decreased $N$ from 7 to 5 . We then made the inverse transform of the cleaned FFT and added the result to the large-scale emission and point source maps. 

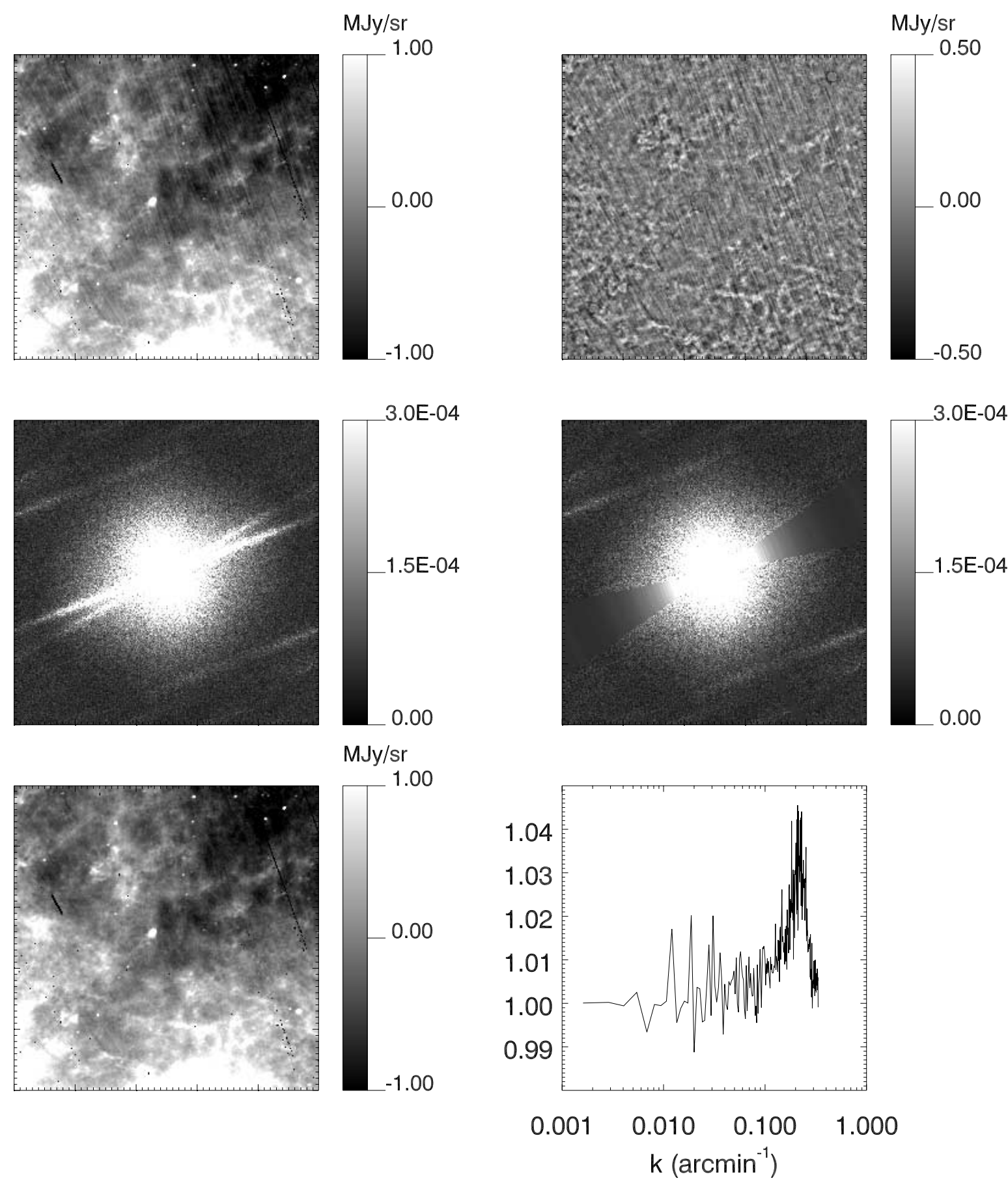

FIG. 1.-Illustration of the destriping algorithm. The image (plate number 83 at $60 \mu \mathrm{m}$ ) is chosen such that it contains point sources, diffuse emission, and undefined regions. Top left: HCON; top right: wavelet filtered HCON; middle left: FFT of the filtered HCON; middle right: modified FFT; bottom left: destriped HCON; bottom right: ratio of the power spectrum of the HCON and the destriped HCON.

This procedure can only be applied on fully defined HCONs. For each plate, the missing data were iteratively replaced by the destriped data of the companion HCONs. When large regions have not been observed by the three HCONs, we used the DIRBE data, corrected for the appropriate gain and offset values.

Our procedure has been extensively tested on simulations. We simulated typical interstellar diffuse regions using fractional Brownian motion images (Miville-Deschênes et al. 2003) with a similar power spectrum to what is observed in the ISM (spectral index of -3 ; Gautier et al. 1992). We added to this map typical random noise together with stripes that were simulated in Fourier space (note that we cannot inject in our simulations empirically derived stripes from real data since such maps, obtained by wavelet filtering real data visually dominated by stripes, are also contaminated by the instrumental noise). The simulated map were then processed through the whole destriping procedure. An example of such a simulated map for a typical high-latitude region at $12 \mu \mathrm{m}$ (where the amplitude of the stripes are of the same order than the signal) is shown on Figure 2, together with the result of the destriping algorithm. We computed the power spectrum of the input noisy interstellar map and that of the recovered map. In such regions, we modified the power spectrum at small scales by only about $3 \%$. This demonstrates that we have suppressed the contribution of the stripes (that was 4 times higher 


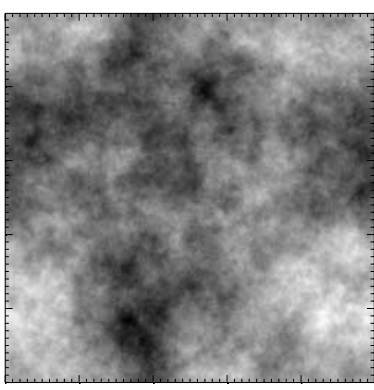
1.00
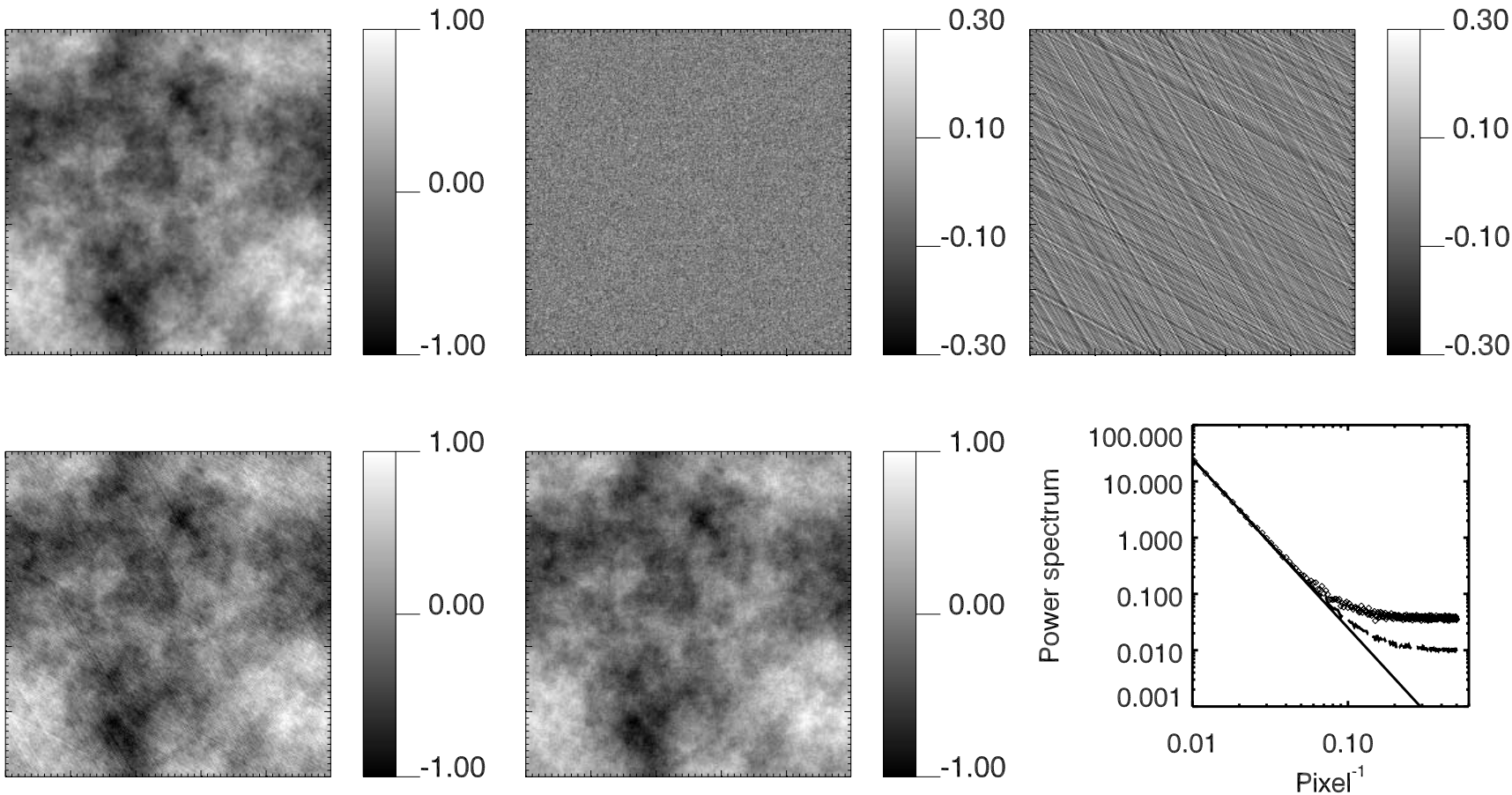

FIG. 2.-Simulated high-latitude cirrus region with noise and stripes. Top left: cirrus $\left[P(k) \propto k^{-3}\right]$; top middle: random noise; top right: stripes; bottom left: total

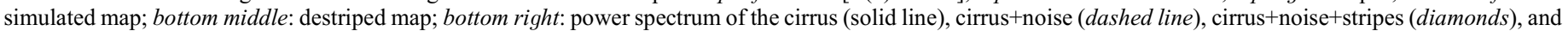

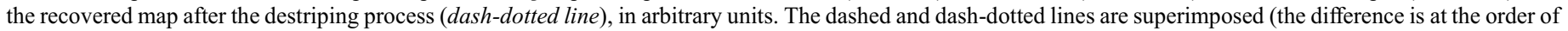
$3 \%$ at very small scales).

than the signal on the power spectrum at small scales in this case) without adding extra noise. In brighter regions, the contribution of the stripes becomes smaller compared to the Galactic emission, leading to a still better recovery of the simulated map. These simulations clearly show the robustness and efficiency of our destriping process.

\section{CALIBRATING THE ISSA PLATES}

\subsection{Infrared Photodetectors}

Wheelock et al. (1993) showed that the responsivity of all $I R A S$ detectors are affected by memory effects that introduce a calibration difference between diffuse emission and point sources. Since IRAS, and especially with the ISO mission, several studies have been done to understand the response of infrared photo conductors like the IRAS 60 and $100 \mu \mathrm{m} \mathrm{Ge:Ga} \mathrm{detectors.}$ Using solid state and semiconductor physics, Vinokurov \& Fouks (1991) and Fouks \& Schubert (1995) have studied the response of such detectors. They showed that the instantaneous response depends on the illumination and on the history of the detector. A simplify version of their model was successfully applied to ISOCAM observations (Coulais \& Abergel 2000). This nonlinear model is rather complex, and it is not the purpose of the present paper to describe it in detail. But to feel how the response of infrared photo detectors behaves, let us consider the simple case of a detector stabilized at flux $J_{0}$ that is suddenly illuminated at flux $J_{1}$ at time $t=0$. In this case, the response $J(t)$ of the detector as a function of time is described by

$$
J(t)=\beta J_{1}+\frac{(1-\beta) J_{1} J_{0}}{J_{0}+\left(J_{1}-J_{0}\right) \exp \left(-t J_{1} / \lambda\right)} .
$$

The $\beta$ and $\lambda$ parameters depends on the physical properties of the detector. An example of this model is shown in Figure 3, where we illustrate what would be the response of a detector when the flux is double $\left(J_{1}=2 J_{0}\right)$, for three values of the initial flux $J_{0}$. We see that the response of the detector is very sensitive to the flux value; for a lower flux value, the detector takes more time to stabilize. Therefore, for a given integration time, an intensity fluctuation on a low surface brightness region will be systematically underestimated in comparison to a brighter one.

This has an important consequence on survey observations like the IRAS mission that scanned the sky with a fixed

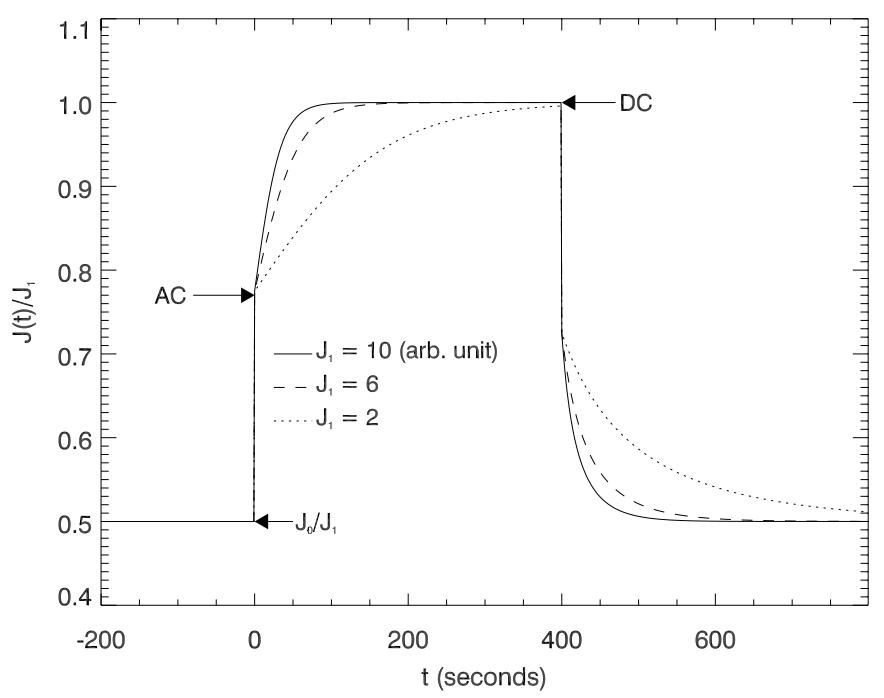

FIG. 3.-Illustration of the response of an infrared photo detector based on eq. (2) (Fouks \& Schubert 1995). The response to upward and downward flux steps are shown for three values of the flux $J_{1}=2,6$, and 10 . To illustrate the behavior of the detector when the flux value is double, the lower flux value is $J_{1} / 2$ for each case. The response of the detector is very sensitive to the flux value; for a lower flux value, the detector takes more time to stabilize. 
TABLE 2

RESPONSIVITY FACTORS

\begin{tabular}{|c|c|c|c|c|c|c|c|c|}
\hline \multirow{2}{*}{$\begin{array}{c}l \\
(\mathrm{deg})\end{array}$} & \multirow[b]{2}{*}{$12 \mu \mathrm{m} R_{0}$} & \multirow[b]{2}{*}{$25 \mu \mathrm{m} R_{0}$} & \multicolumn{3}{|c|}{$60 \mu \mathrm{m}$} & \multicolumn{3}{|c|}{$100 \mu \mathrm{m}$} \\
\hline & & & $R_{0}$ & $A$ & $B$ & $R_{0}$ & $A$ & $B$ \\
\hline $0.067 \ldots \ldots \ldots \ldots \ldots \ldots \ldots$ & 0.78 & 0.82 & 0.92 & 0.0 & 0.0 & 1.0 & 0.0 & 0.0 \\
\hline 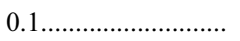 & 0.90 & 0.90 & & & & & & \\
\hline $0.2 \ldots \ldots \ldots \ldots \ldots \ldots$ & 0.92 & 0.93 & & & & & & \\
\hline $0.5 \ldots \ldots \ldots \ldots \ldots \ldots \ldots$ & 0.98 & 0.97 & & & & & & \\
\hline $1.0 \ldots \ldots \ldots \ldots \ldots \ldots \ldots$ & 1.00 & 0.98 & & & & & & \\
\hline $1.25 \ldots \ldots \ldots \ldots \ldots \ldots$ & & & 1.00 & 0.08 & $-5.0 \mathrm{E}-5$ & 0.96 & 0.008 & $-4.8 \mathrm{E}-5$ \\
\hline $1.5 \ldots \ldots+\ldots$ & 1.00 & 1.00 & & & & & & \\
\hline $2.0 \ldots \ldots \ldots \ldots \ldots \ldots \ldots$ & 1.00 & 1.00 & & & & & & \\
\hline $2.5 \ldots \ldots$ & & & 1.01 & 0.01 & $-4.0 \mathrm{E}-5$ & 0.99 & 0.011 & $-6.5 \mathrm{E}-5$ \\
\hline $5.0 \ldots \ldots \ldots \ldots \ldots \ldots \ldots .$. & & & 1.05 & 0.014 & $-6.0 \mathrm{E}-5$ & 1.08 & 0.012 & $-8.0 \mathrm{E}-5$ \\
\hline 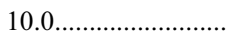 & & & 1.13 & 0.014 & $-9.0 \mathrm{E}-5$ & 1.25 & 0.08 & $-5.9 \mathrm{E}-5$ \\
\hline
\end{tabular}

Notes.-The responsivity $R$ at a given wavelength, scale and brightness is given by eq. (3). The responsivity at 12 and $25 \mu \mathrm{m}$ does not depend on brightness $(A=0, B=0)$. The value of the responsivity at the smallest scale $\left(0^{\circ} 067\right)$ is the DC/AC factor (see Table 1$)$ needed to recover the correct point source calibration.

integration time. It is likely that, at low flux value, the integration time was shorter than the stabilization time of the detector. Therefore, the amplitude of the fluctuations on the sky are systematically underestimated in faint regions. This type of memory effect should leave another signature on maps with a fixed integration time. As the satellite scans the sky it observes structures with various angular scales. These structures can be seen as flux steps like in Figure 3. Then, when the satellite goes through a small-scale structure, it is likely that the observed flux will never reach the stabilization level. On the other hand, for very large-scale structures, the detector has enough time to stabilize. Therefore, the amplitude of the intensity fluctuations are underestimated at low flux but also at small scales.

\subsection{Responsivity of the 12 and $25 \mu \mathrm{m}$ Detectors}

Similar effects were indeed observed by the IRAS team. By staring at several stars of different brightness, Wheelock et al. (1993) showed that the responsivity $R$ of the 12 and $25 \mu \mathrm{m}$ detectors depends on time and therefore on angular scale, as the satellite is scanning the sky at a fixed rate $\left(3 .^{\prime} 85 \mathrm{~s}^{-1}\right)$. The responsivity was observed to be constant for angular scales larger than $2^{\circ}$ but it decreased at smaller scales, in accordance with the model described earlier. On the other hand the IRAS team reports no variations of the responsivity as a function of brightness for these detectors. In Table 2 we give the responsivity variation with angular scale from Wheelock et al. (1993) for the 12 and $25 \mu \mathrm{m}$ detectors, but one must be aware that this variation of the responsivity with scale was not applied to the ISSA plates. Instead a constant responsivity factor was used to match the responsivity at large scales (i.e., the ISSA plates are DC calibrated), which means that the brightness fluctuations at scales smaller than $\sim 2^{\circ}$ are systematically underestimated at 12 and $25 \mu \mathrm{m}$.

\subsection{Responsivity of the 60 and $100 \mu \mathrm{m}$ Detectors}

At 60 and $100 \mu \mathrm{m}$ the situation is complicated by the fact that the responsivity depends both on time (i.e., angular scale) and brightness. Wheelock et al. (1993) did not give any prescription to address this problem. To evaluate the variation of the responsivity with scale and brightness of the 60 and $100 \mu \mathrm{m}$ detectors we compared the IRAS and DIRBE data using a wavelet analysis.

To study the variations of the 60 and $100 \mu \mathrm{m}$ responsivity we have looked at variations of $\triangle I R A S / \triangle D I R B E$ as a function of scale and brightness. For a given diffuse structure on the sky the quantity $\triangle I R A S / \triangle \mathrm{DIRBE}$ represents the ratio of the brightness fluctuation (above the background) of that structure seen in the $I R A S$ and DIRBE data. If we take the DIRBE calibration as an absolute reference, the quantity $\triangle I R A S / \triangle \mathrm{DIRBE}$ is equal to the $I R A S$ responsivity $R$ to a brightness fluctuation. To compute $\triangle I R A S / \triangle \mathrm{DIRBE}$ as a function of scale and brightness we first put the two data set on a common grid and at the same angular resolution. For each ISSA plate we have constructed a larger map $(600 \times 600$ pixels $)$ by mosaicking neighboring plates. This step is necessary in order to avoid edge effects in further convolutions. Next this bigger ISSA plate is convolved by the DIRBE beam. The DIRBE data are then projected on the ISSA grid. Finally, both maps are convolved by a Gaussian beam of FHWM $40^{\prime}$ to smooth out the DIRBE pixelization (Schlegel et al. 1998) and the extra 50 pixels wide edges are removed. At that point the angular resolution and size of both maps are of $1^{\circ}$ and $500 \times 500$, respectively.

To compute the variation of $\triangle I R A S / \triangle \mathrm{DIRBE}$ with scale and brightness we used a wavelet transform (the "a trou" algorithm; Starck \& Murtagh 1998) that allows us to study the brightness variations as a function of scale. An example of such a decomposition is shown in Figure 4 on a fractional Brownian motion (fBm) image (Miville-Deschênes et al. 2003) with a similar power spectrum to what is observed in the ISM (spectral index of -3 ) and convolved the same way the IRAS and DIRBE data were. On the top row of Figure 4 we show two $\mathrm{fBm}$ images, $m_{1}$ and $m_{2}$, where $m_{2}$ was constructed by simply applying a constant multiplicative factor to $m_{1}\left(m_{2}=Q m_{1}\right)$. On the four bottom rows we show the wavelet coefficients at four different scales $\left(1.25,2^{\circ} .5,5^{\circ}\right.$, and $\left.10^{\circ}\right)$. The important result here is that the slope of the linear regression of the wavelet coefficients gives exactly the multiplicative factor $Q$ at each scale.

An example of the same wavelet decomposition for a typical $60 \mu \mathrm{m}$ field is shown in Figure 5. One would notice that the wavelet coefficients of the IRAS and DIRBE are very well fitted by a linear fit with relatively small dispersion. One noteworthy result here is the increase with scale of the slope $(\triangle I R A S / \triangle \mathrm{DIRBE})$ of the linear regression between DIRBE and IRAS data. The trend seen in Figure 5 is totally representative of what is generally observed.

We have performed this analysis at 60 and $100 \mu \mathrm{m}$, on all the ISSA plates with no undefined values, to also study the 

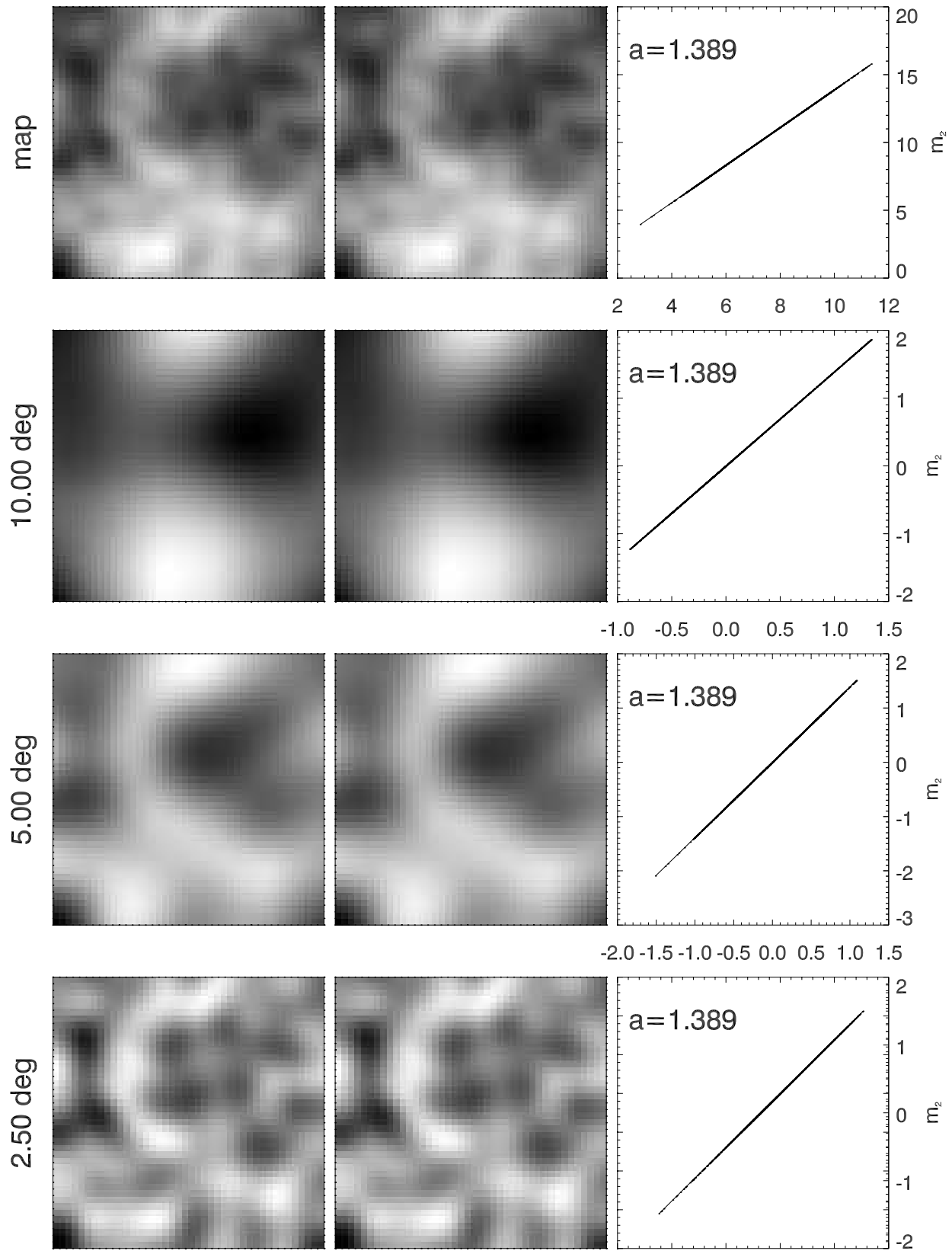

$-2.0-1.5-1.0-0.50 .00 .51 .01 .5$
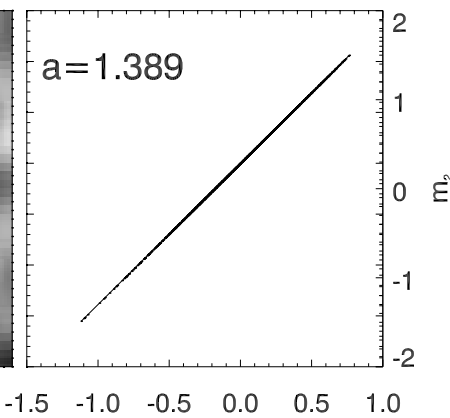

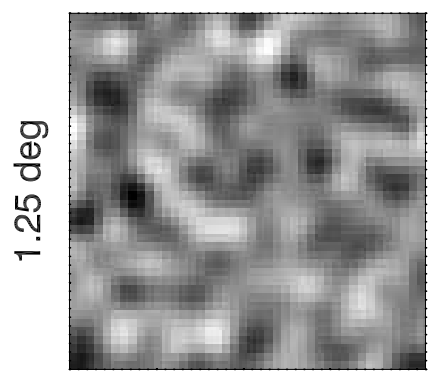

$\mathrm{m}_{1}$

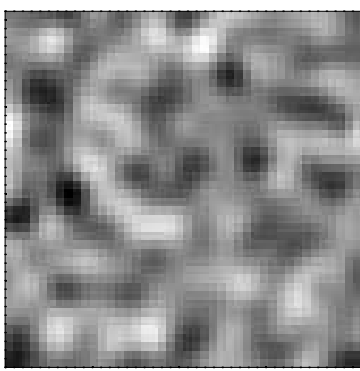

$\mathrm{m}_{2}$

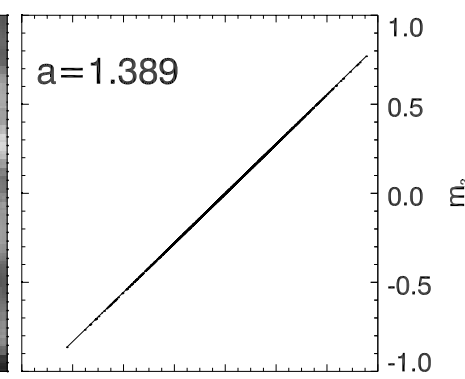

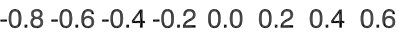

$\mathrm{m}$

FIG. 4. - Illustration of the use of the wavelet decomposition to compute a gain difference between two maps at different scales. Here the $m_{1}$ and $m_{2}$ are identical fBm images (shown in the top row) with a constant multiplicative factor between the two $\left(m_{2}=1.389 m_{1}\right)$. The four bottom rows show the wavelet decomposition of $m_{1}$ and $m_{2}$. The right column shows the correlation between $m_{2}$ and $m_{1}$ for the whole map and at each scale. The slope $a$ of the linear regression is given in each panel. 

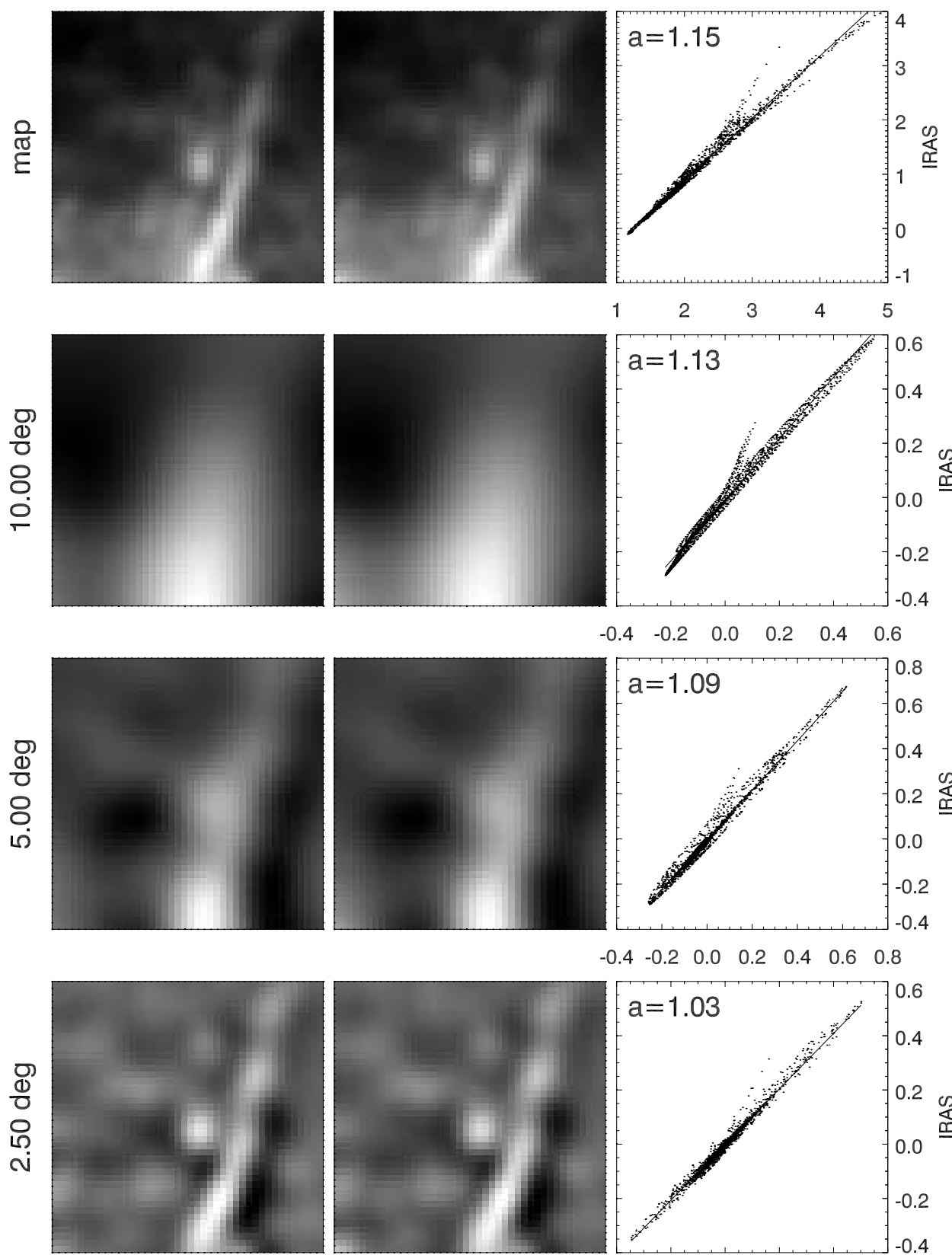

$\begin{array}{lllllll}-0.4 & -0.2 & 0.0 & 0.2 & 0.4 & 0.6 & 0.8\end{array}$

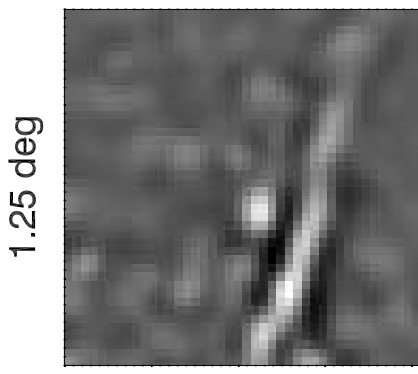

DIRBE
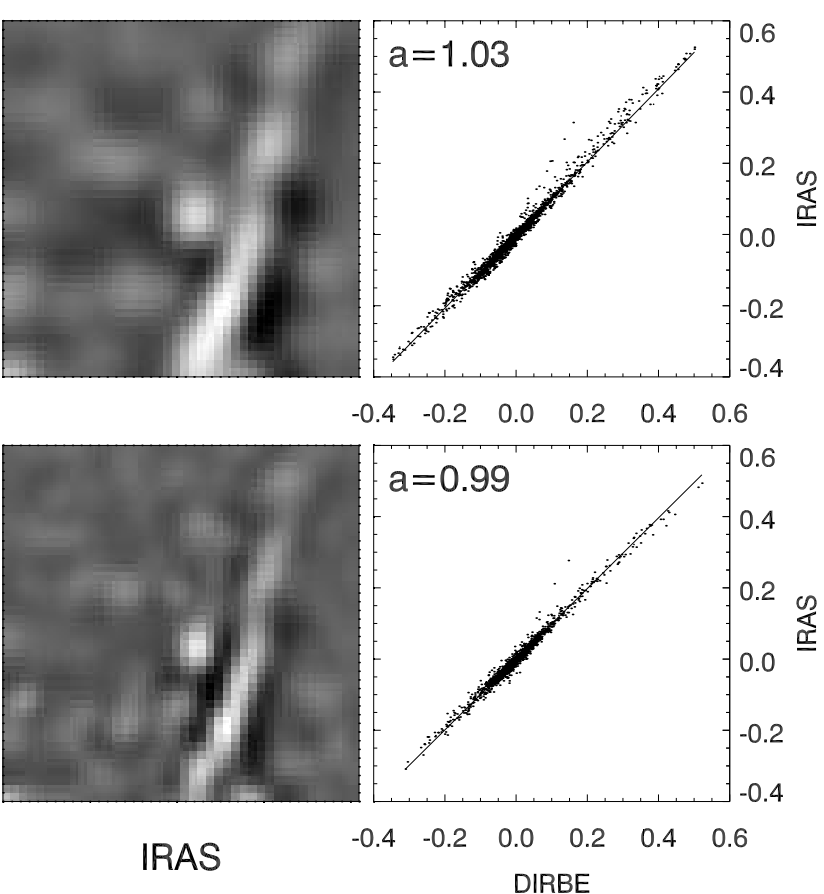

FIG. 5.-Example of a wavelet decomposition of a typical $60 \mu \mathrm{m}$ image. See Fig. 4 for details. 


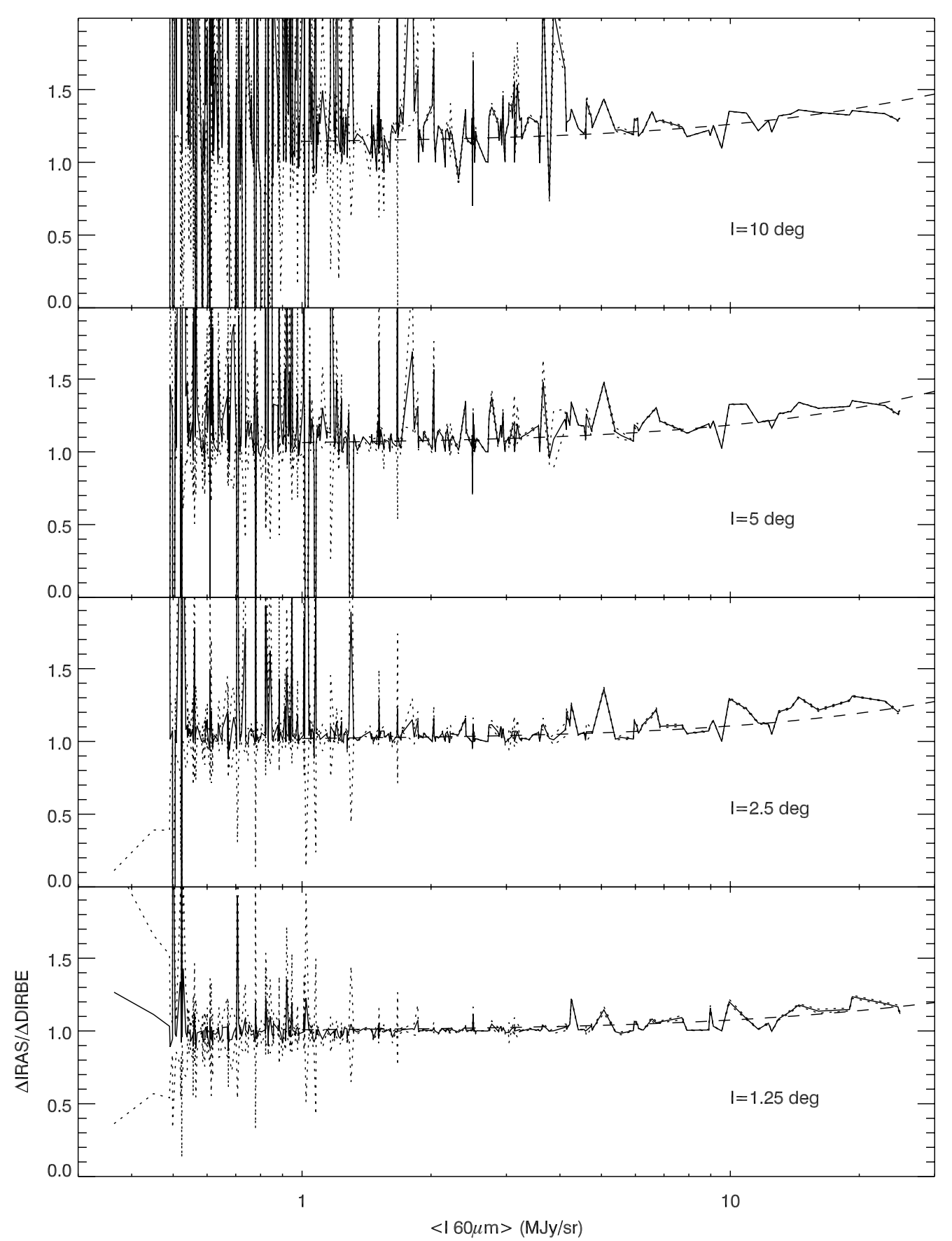

FIG. 6.-Responsivity of the $60 \mu \mathrm{m} I R A S$ data compared to DIRBE as a function of the $60 \mu \mathrm{m}$ DIRBE brightness and for four different scales. The dotted lines are the slope of the linear regression of IRAS vs. DIRBE and DIRBE vs. IRAS. The solid line is the average of the two dotted lines. The dashed line is the fit to the average slope using eq. (3).

variation of $R=\Delta I R A S / \triangle \mathrm{DIRBE}$ with brightness $\left\langle I_{\lambda}\right\rangle^{3}{ }^{3}$ The results of this analysis are compiled in Figures 6 and 7 , where we show the variation of $R$ with brightness at the four scales studied. From these two figures we found that the IRAS responsivity $R$ increases with brightness for the two bands. The increase of $R$ with scale described previously is also seen in these two figures. In addition, the values of $R$ are systematically above 1.0 at large scales, which confirms that IRAS brightness fluctuations are overestimated at large scales in the ISSA plates. The values obtained here at scales of $10^{\circ}$ and for brightness of

\footnotetext{
${ }^{3}$ Note here that, as stated at the end of this section, $\left\langle I_{\lambda}\right\rangle$ is the median value of the DIRBE data to limit the effect of variations of the IRAS zero level.
}

$\sim 3$ and $\sim 10 \mathrm{MJy} \mathrm{sr}^{-1}$ at 60 and $100 \mu \mathrm{m}$ are compatible with the IRAS-DIRBE correction factors given by Wheelock et al. (1993) (see Table 1).

The increase of $R$ with scale and brightness indicates that the IRAS responsivity is generally lower for small-scale and faint brightness fluctuations than for large-scale and large brightness fluctuations. This behavior is in complete accordance with what is expected for typical photoconductors (see $\S 4.1$ ).

The dotted lines in Figures 6 and 7 represent the slope of the linear regression of IRAS versus DIRBE and DIRBE versus IRAS, and the solid line is the average of the two. Computing both linear regressions allows us to conclude that the trend observed here is not due to noise. The effect of detector noise is 


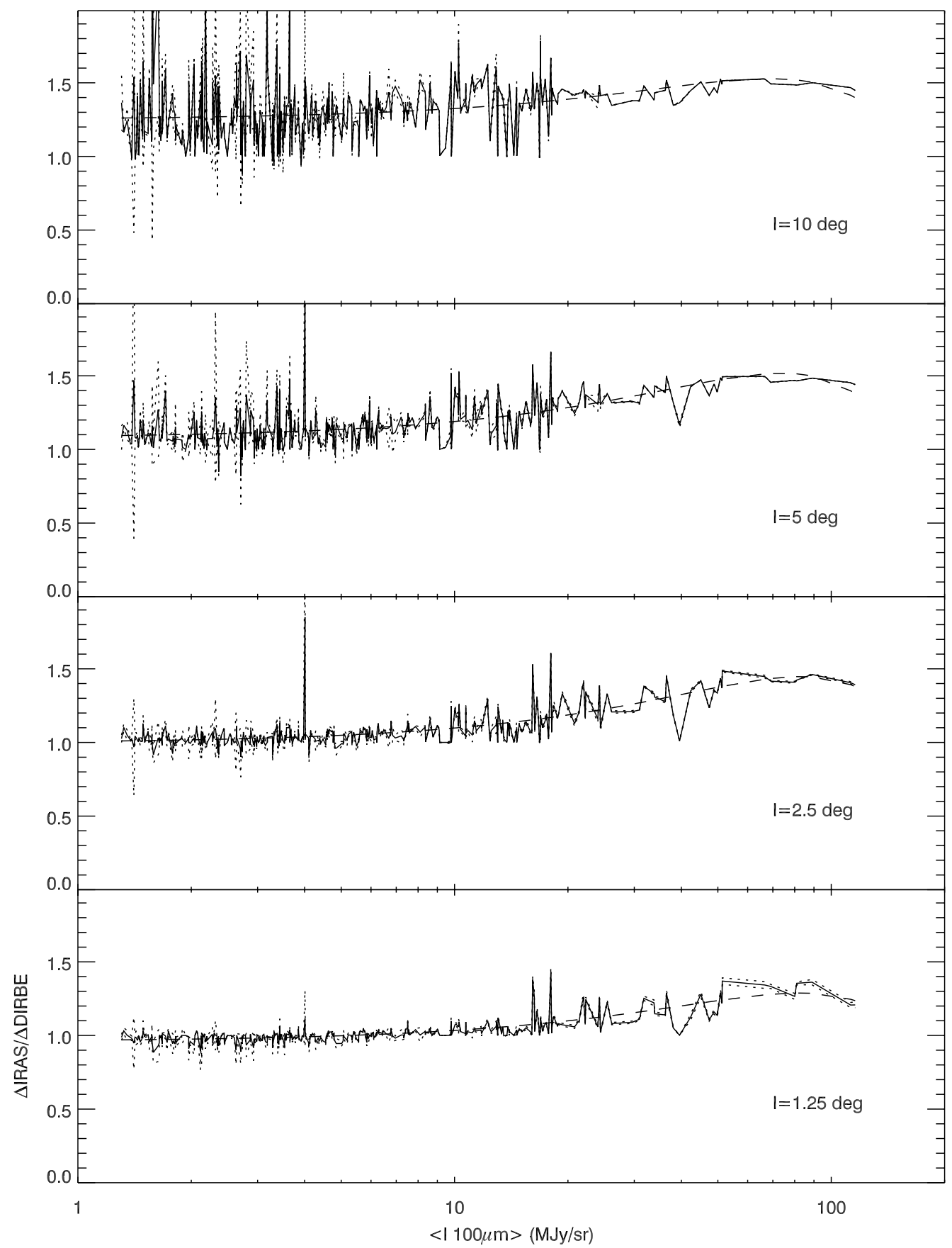

FIG. 7.-Responsivity of the $100 \mu \mathrm{m}$ IRAS data compared to DIRBE as a function of the $100 \mu \mathrm{m}$ DIRBE brightness and for four different scales. See Fig. 6 for details.

clearly seen at small scale and at low brightness, where the dispersion of the slope values is increasing. There is also an increase of the dispersion at large scales (very clear at $60 \mu \mathrm{m}$ ), caused by the large-scale drift of the detector responsivity or to a bad zodiacal light correction. These two effects produce an additive effect with spatial variations at scales larger than a few degrees, affecting predominantly the slope measured at larger scales and at low brightness.

The curves in Figures 6 and 7 can be reasonably well fitted by the following function:

$$
R=R_{0}+A\left\langle I_{\lambda}\right\rangle+B\left\langle I_{\lambda}\right\rangle^{2} .
$$

The values of $R_{0}, A$, and $B$, which all depend on $\lambda$ and scale $l$, are given in Table 2 . The brightness $\left\langle I_{\lambda}\right\rangle$ is the median value of the DIRBE data, to limit the effect of variations of the IRAS zero level.

\subsection{Responsivity Correction}

In the previous sections we showed that the IRAS responsivity to diffuse emission varies with scale and, at 60 and $100 \mu \mathrm{m}$, also with brightness. At these wavelengths we were able to establish an analytical form of the responsivity variations (eq. [3]) by comparing the IRAS and DIRBE data. This expression is also valid for the 12 and $25 \mu \mathrm{m}$ bands; in this case it reduces to $R=R_{0}$ as the responsivity does not depend on the brightness $(A=0$ and $B=0)$.

The responsivity correction to apply to the ISSA plates is the inverse of the expression given in equation (3) $(G=1 / R)$. In 
$60 \mu \mathrm{m}$

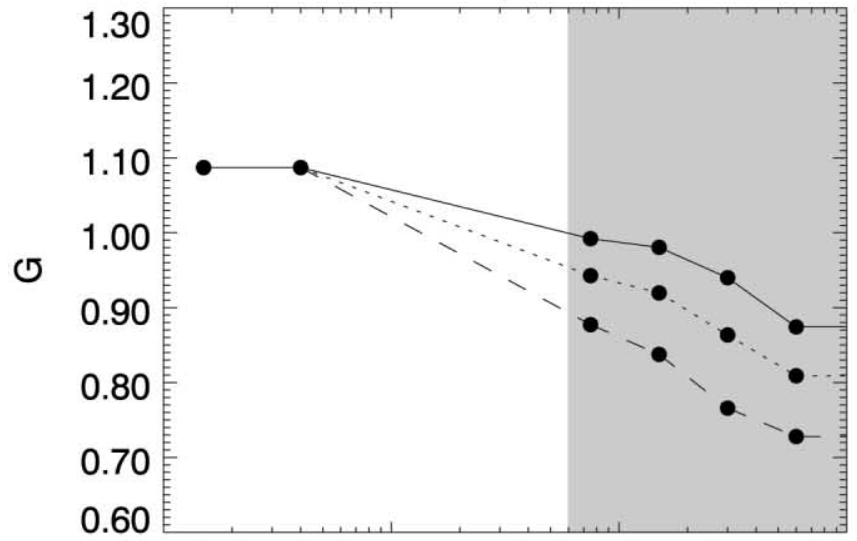

$12 \mu \mathrm{m}$

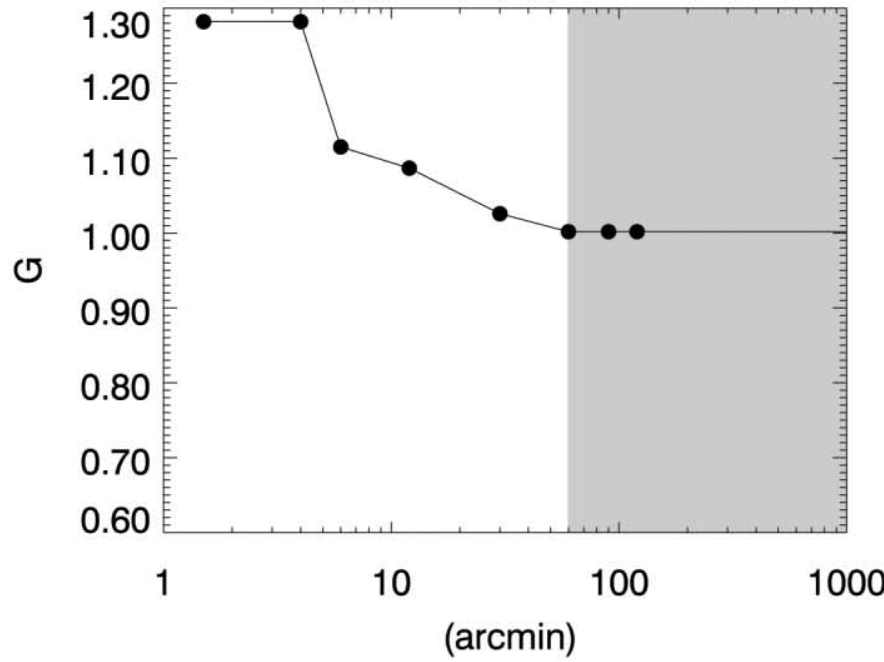

$100 \mu \mathrm{m}$

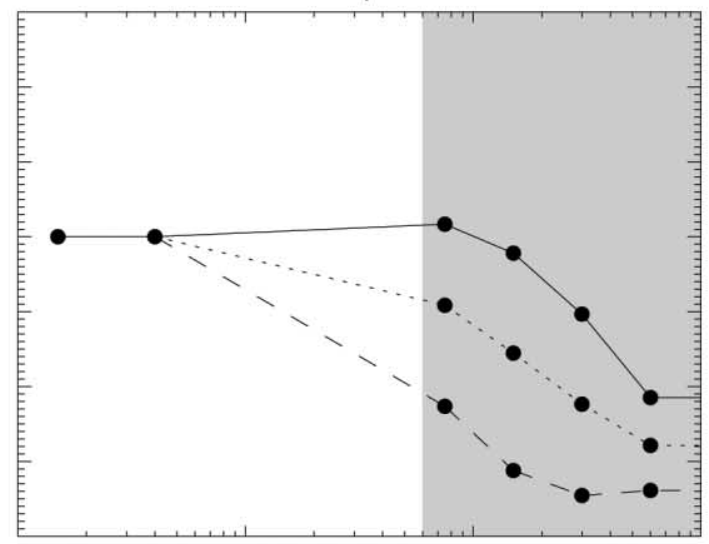

$25 \mu \mathrm{m}$

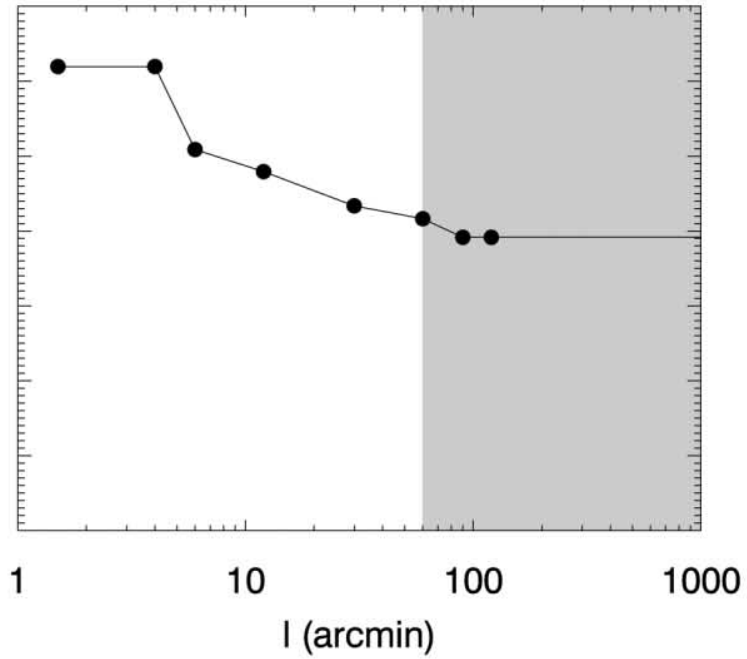

FIG. 8.- Responsivity correction factor $(G=1 / R)$ for the four bands as a function of angular scale. For the 60 and $100 \mu \mathrm{m}$ bands the gain factor is given for three

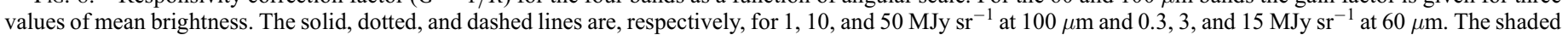
gray area represents the angular scales on which DIRBE data are used to scale the IRAS data.

Figure 8 we show the responsivity correction factor as a function of scale for the four bands. For the 60 and $100 \mu \mathrm{m}$ bands we present three curves to show the variation of the responsivity correction with brightness.

The implementation of such a correction is made difficult by the fact that it depends on brightness (at 60 and $100 \mu \mathrm{m}$ ). We need to apply a correction that depend on scale but also on position to take into account brightness variations across the map. To achieve this we use a local approach based on a wavelet decomposition of each ISSA HCON.

To avoid discontinuity problems between adjacent plates each $\mathrm{HCON}$ is put in a $600 \times 600 \mathrm{map}$ and the undefined regions are filled using other HCONs or, when IRAS data are unavailable, by DIRBE (properly scaled to take into account the gain difference between the two data sets). For each HCON at each wavelength, we first separated point sources from diffuse emission (see Appendix A). To correct the diffuse emission map we decomposed it (using the "a trou" algorithm) for the first six scales (corresponding to $3^{\prime}, 6^{\prime}, 12^{\prime}, 24^{\prime}, 48^{\prime}, 1^{\circ} .6$, and $3^{\circ} .2$ ). Then we computed the proper gain correction factor at each scale and for each pixel of the map using equation (3). To compute this correction at 12 and $25 \mu \mathrm{m}$ bands we simply use the responsivity values given in Table 2 and interpolate at the six corresponding scales (from $3^{\prime}$ to $3^{\circ} .2$ ). At the 60 and $100 \mu \mathrm{m}$ we do the same but we also use the DIRBE brightness to compute the brightness dependence part of the responsivity correction.

The wavelet coefficients of the diffuse emission map were then recombined to form the responsivity corrected diffuse emission map. Finally, the point sources map, properly calibrated by applying the corresponding DC/AC factor (see Table 1) was added to the corrected diffuse emission map. The responsivity correction applied here does not correct the emission at scales larger than $3^{\circ} .2$. We take care of this in the next and final step of the processing.

\subsection{Zero Level Correction}

As it was mentioned earlier the zero level of the IRAS data is not well determined, owing to detector drifts and/or residual zodiacal emission. To correct this large-scale responsivity and the spatially varying zero level of the ISSA maps we again used the DIRBE data that were better calibrated and that used a better zodiacal emission model. Here we make the assumption that the zero level does not vary on scales smaller than $1^{\circ}$, which is reasonable as detector drifts and zodiacal emission are not expected to vary on such scales.

As for the responsivity correction we computed the offset map for every HCON, enlarged to $600 \times 600$ to make sure that we keep the continuity between adjacent ISSA plates. Empty 
pixels were filled with the average map (HCON0), previously corrected for the responsivity variation, or DIRBE data when necessary. The HCON map was then convolved by the DIRBE beam and by a Gaussian beam (FWHM $\left.=40^{\prime}\right)$ to smooth out the DIRBE pixelization. The DIRBE data were projected on this enlarged HCON map and convolved by the same Gaussian beam. At that point both data sets are on the same grid and at a $1^{\circ}$ resolution. ${ }^{4} \mathrm{We}$ then computed the difference between the two maps (DIRBE-IRAS). This difference map was added to the HCON. When all the three HCONs were corrected we computed the average map (HCON-0) using the appropriate coverage maps. This is the final step of the processing.

\section{PROPERTIES OF THE IRIS PLATES}

The processing of the $4893 \mathrm{HCON}$ plates was done using the McKenzie cluster of the Canadian Institute for Theoretical Astrophysics. This Beowulf cluster is composed of $5122.4 \mathrm{GHz}$ Xeon processors with a total of 256 GB of RAM. We effectively used only 40 CPUs of the cluster at the same time and the total computation time for the whole processing (deglitching, destriping, responsivity and offset correction) was $\sim 20$ hours.

Figures 9, 10, 11, and 12 present the ISSA and IRIS plate number 81 at the four wavelengths. Are also shown the difference between the ISSA and IRIS maps and the ratio of their power spectra. These figures show that the destriping described in $\S 3.2$ removed very efficiently the stripes at all wavelengths. The second more noticeable improvement is the modification of large-scale structures in each maps, due to the responsivity and zero level corrections. These two corrections removed residual zodiacal light and detector drifts very well and significantly improved the calibration of the data. The correction of the largescale structures of the maps (that is due both to responsivity and zero level corrections) is clearly seen in the ratio of the power spectra.

One interesting property seen on Figures 9 and 10 is that, even after the destriping, the power spectra ratio of the 12 and $25 \mu \mathrm{m}$ images is $\sim 1$ on scales between $5^{\prime}$ and $1^{\circ}$. This is due to the responsivity correction applied (see Fig. 8) that is higher than 1.0 at those scales. The noise reduction resulting in improved signal-to-noise ratios obtained from the destriping is almost counterbalanced by the multiplication of all the smallscale fluctuations (including noise) by a factor greater than 1 . Nevertheless, the noise properties of the IRIS plates are much more easy to handle as they are now homogeneous. On the other hand, at 60 and $100 \mu \mathrm{m}$ the responsivity correction is not as important and there is a noise reduction that is about $15 \%-20 \%$ at scales $5^{\prime}-15^{\prime}$.

Finally, notice that the areas of the sky not covered by the IRAS survey are left empty in the IRIS product.

\subsection{Noise}

Following the method described by Miville-Deschênes et al. (2002b) one can estimate the power spectrum of the noise of each IRIS map. To do so we take advantage of the fact that each final IRIS map is composed of a combination of up to three HCONs. Here we argue that the difference between two HCONs

\footnotetext{
${ }^{4}$ It is worth mentioning that point sources were removed in the IRAS and DIRBE data to compute the offset. Even after the gain correction, which reconciliate the calibration of point sources and diffuse emission, we had to remove point sources for the offset computation as bright sources at 12 and $25 \mu \mathrm{m}$ have relatively strong tails that increase significantly their flux when convolved by the DIRBE beam.
}

is dominated by the instrumental noise and that the power spectrum of such a difference map is equal to the power spectrum of the noise, multiplied by a scaling factor that depends on the coverage maps.

If we make the assumption that the noise level $B$ of the IRAS detectors is stationary, and if we neglect the photon noise (i.e., the noise level is dominated by the readout noise), the noise level at position $(x, y)$ of the $i$ th HCON map is

$$
\sigma_{i}(x, y)=\frac{B}{\sqrt{N_{i}(x, y)}},
$$

where $N_{i}(x, y)$ is the coverage map of the $i$ th HCON map. An example of a typical coverage map is shown on Figure 13.

The noise level of the co-added map (HCON-0) is

$$
\sigma_{0}(x, y)=\frac{B}{\sqrt{N_{\mathrm{tot}}(x, y)}}
$$

where $N_{\text {tot }}(x, y)=N_{1}(x, y)+N_{2}(x, y)+N_{3}(x, y)$. Similarly, the noise level of the difference between two HCON maps $(i$ and $j$ ) is

$$
\begin{aligned}
\sigma_{i-j}(x, y) & =\sqrt{\sigma_{i}^{2}+\sigma_{j}^{2}} \\
& =B \sqrt{\frac{1}{N_{i}(x, y)}+\frac{1}{N_{j}(x, y)}}
\end{aligned}
$$

Combining the last two equations, the noise level of the coadded map becomes

$$
\sigma_{0}(x, y)=\sigma_{i-j} \sqrt{\frac{N_{i}(x, y) N_{j}(x, y)}{N_{\text {tot }}(x, y)\left[N_{i}(x, y)+N_{j}(x, y)\right]}} .
$$

To estimate the power spectrum of the noise of a given IRIS plate we first compute the difference map between two HCONs. The difference map is then normalized following equation (7) to take into account the spatial coverage of every HCON. An example of such a normalized difference map is shown in Figure 13 together with its power spectrum and the power spectrum of the corresponding IRIS plate. At small scales (high $k$ ) these two power spectra meet perfectly, which validates our processing.

It is important to point out that the difference map used here to compute the power spectrum of the noise is by no means a noise level map. It does not provide a way to estimate the noise level of every pixel in the map but it is only used to estimate the global properties of the noise (i.e., power spectrum, average level).

The average noise level of an IRIS map can be estimated by taking the standard deviation of the difference map. Typical noise level values are given in Table 1 for the four bands. Within $10 \%$ the IRIS noise level is the same as the ISSA noise level. This is due to the fact that the power spectrum of the noise is not flat (its slope varies from -1.5 to 0 ). Therefore, the standard deviation of the difference map may be dominated by largescale structures in the noise. In such case, integral of the ISSA and IRIS noise power spectra on selected scales allows a better comparison. For large scales $\left(1^{\circ}-10^{\circ}\right)$, the noise level in the IRIS plates is about $30 \%$ smaller than that of the ISSA plates, 

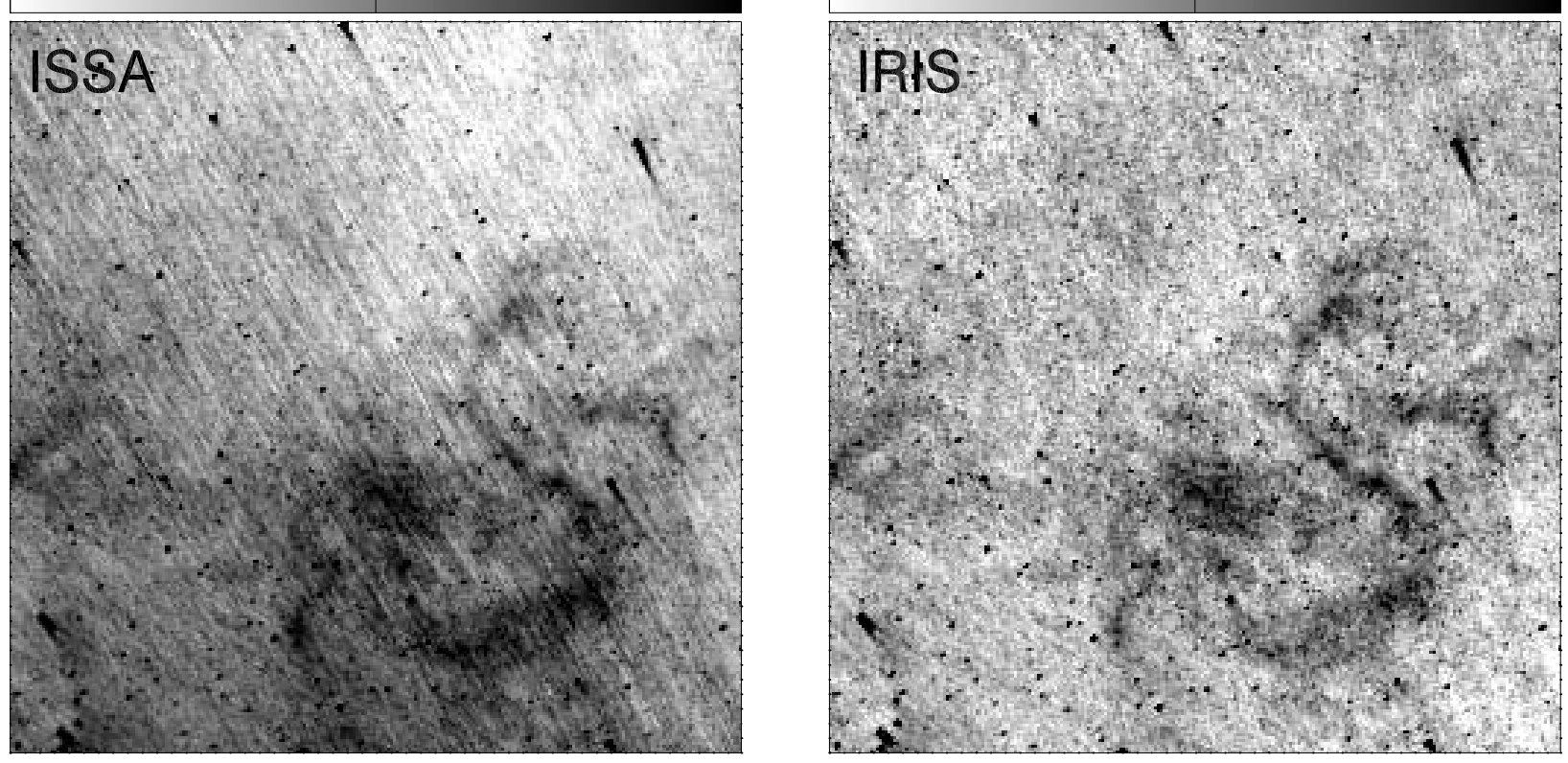

\section{ISSA-IRIS}
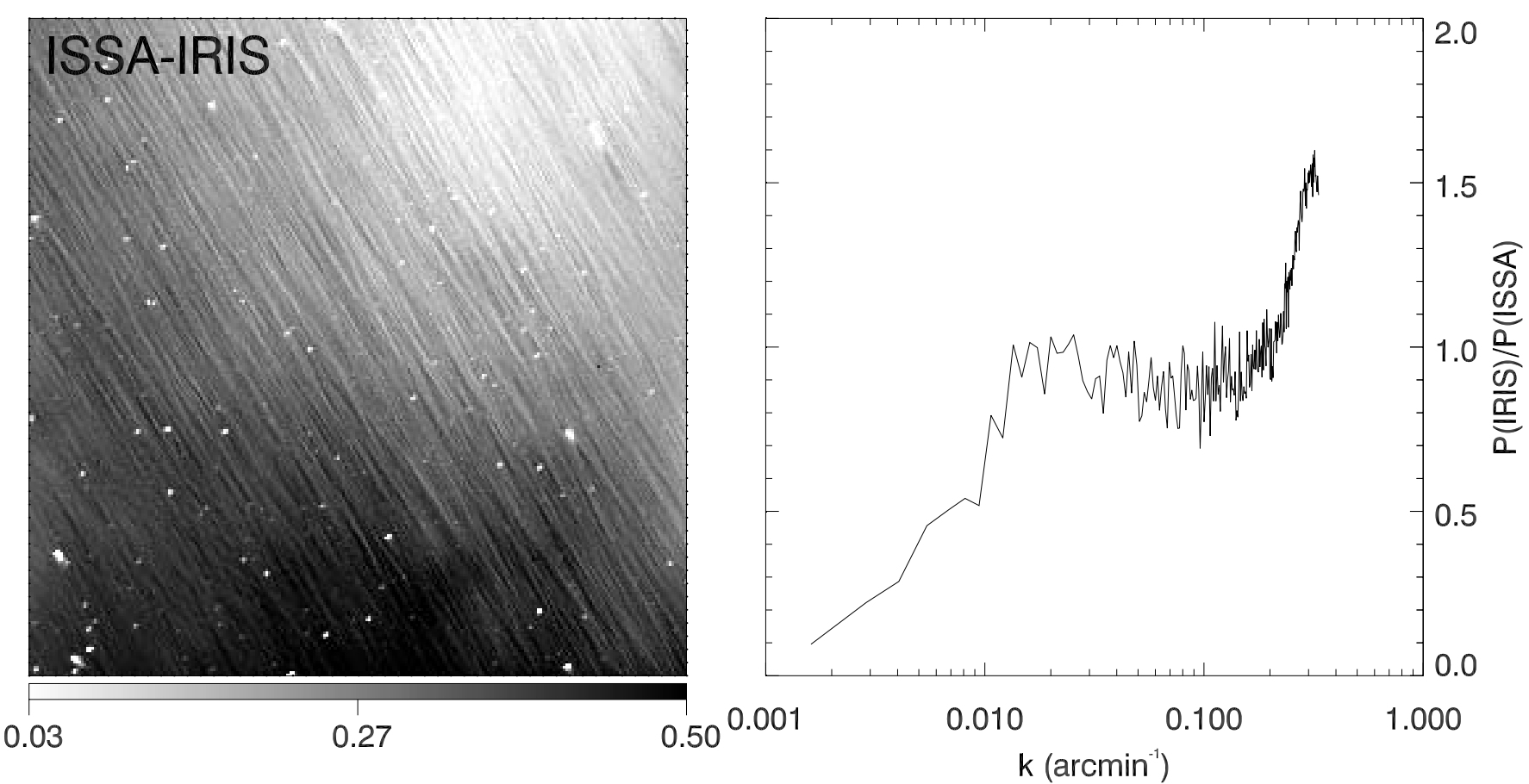

FIG. 9.-Plate number 81 at $12 \mu \mathrm{m}$. Comparison of the ISSA (top left) and IRIS (top right) maps. The difference between the two (ISSA-IRIS) is shown in the bottom left panel. The ratio of the power spectra of the IRIS and ISSA maps is shown in the bottom right panel. 

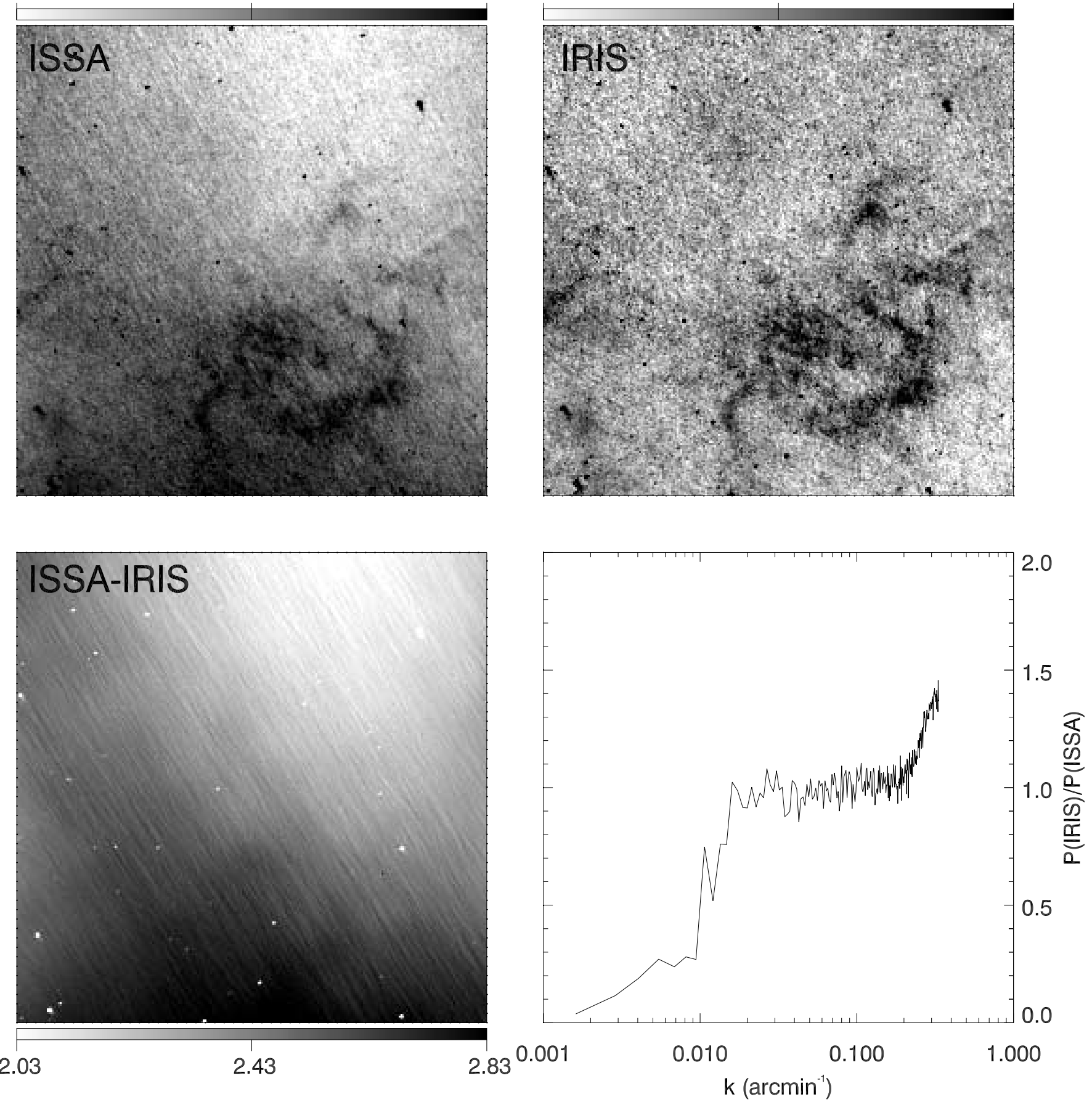

FIG. 10.—Plate number 81 at $25 \mu \mathrm{m}$. See Fig. 9. 


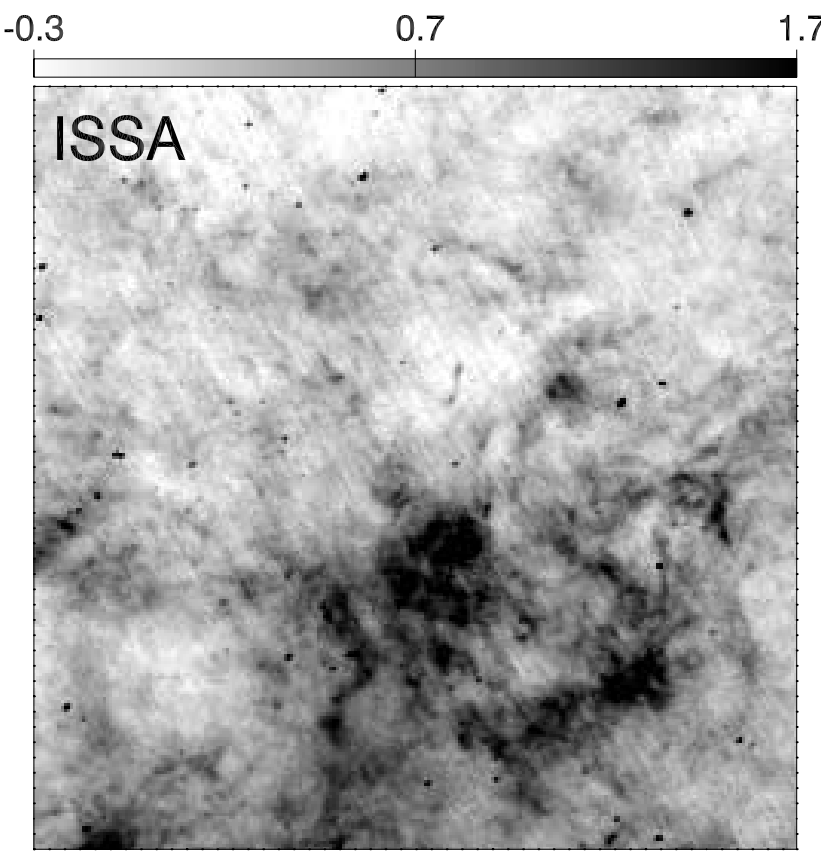

1.1

2.0
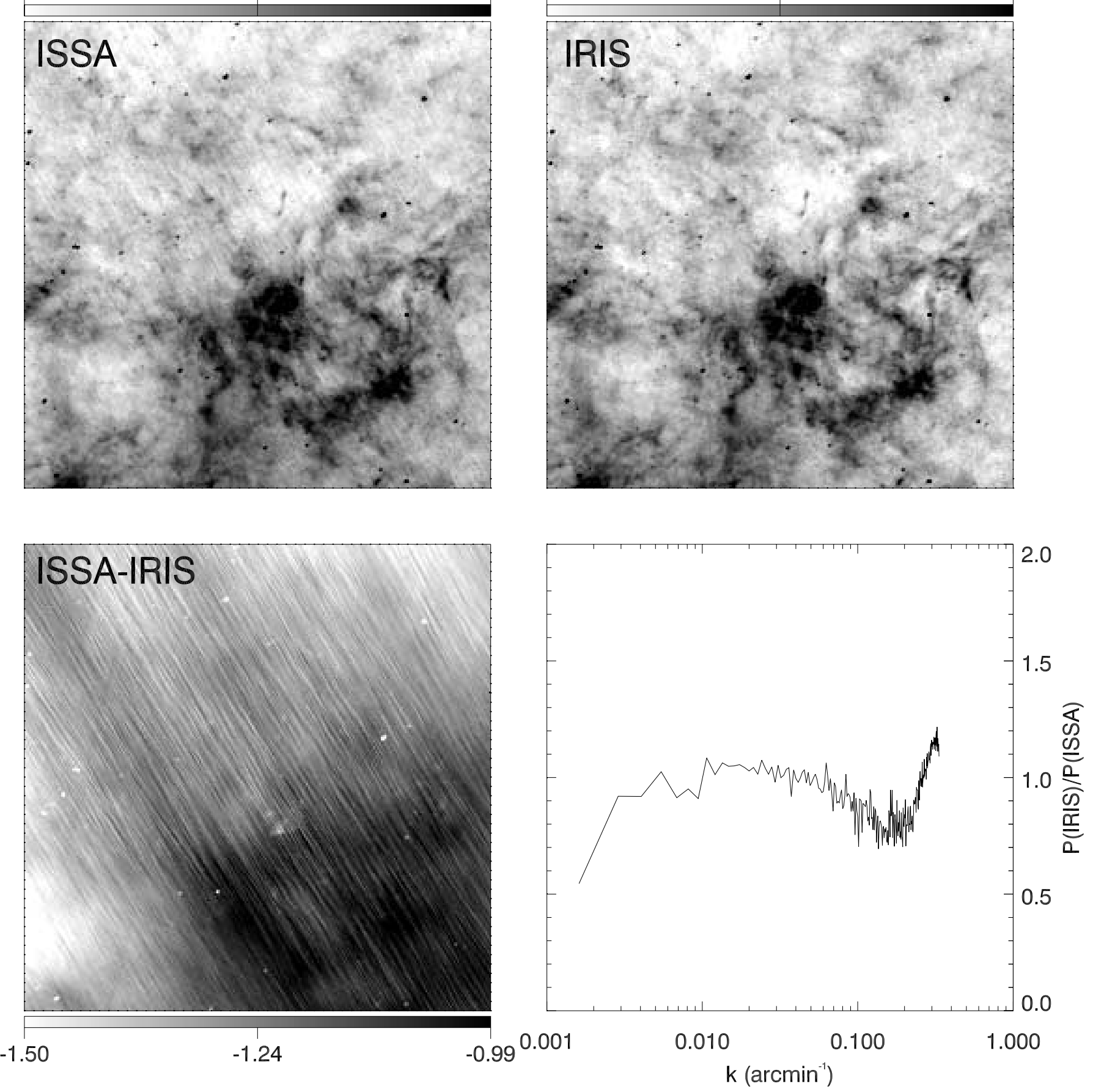

FIG. 11.-Plate number 81 at $60 \mu \mathrm{m}$. See Fig. 9.

for the four wavelengths. At smaller scales, as explained in $\S 5$, we gain in sensitivity only at 60 and $100 \mu \mathrm{m}$. The gain is about $15 \%-20 \%$ for scales $5^{\prime}-15^{\prime}$.

\subsection{Effective Angular Resolution}

Because of the geometry of the IRAS detectors, the IRAS data have a higher spatial resolution in the in-scan direction than in the cross-scan direction (see Table 1). Wheelock et al. (1993) have computed the PSF on few (4 to 5) point sources in each band. They showed that the PSF is noncircular owing to differences in the in-scan and cross-scan resolution. They conclude that the angular resolution of the ISSA plates is between $4^{\prime}$ and $5^{\prime}$ in each band.

Following Miville-Deschênes et al. (2002b) we used the power spectrum of ISSA plates to estimate the effective point spread function (PSF) of the ISSA. If we make the assumption that the effective PSF is described by a Gaussian, the power spectrum of an image dominated by interstellar fluctuations can be expressed by the following equation:

$$
P(k)=\exp \left(\frac{-k^{2}}{2 \sigma_{k}^{2}}\right) \times A k^{\beta},
$$



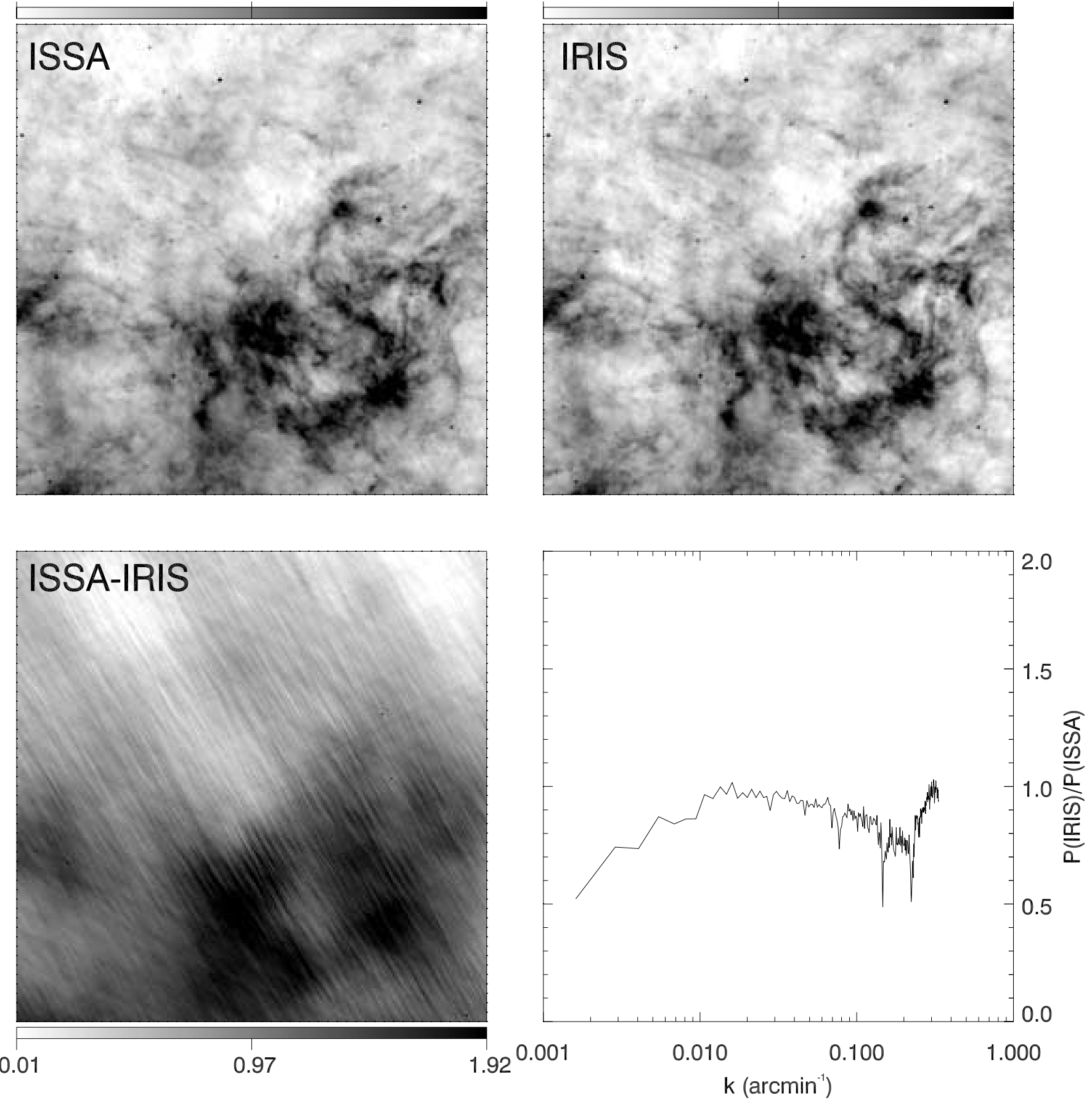

FIG. 12.-Plate number 81 at $100 \mu \mathrm{m}$. See Fig. 9.

where $A k^{\beta}$ describes the cirrus emission power spectrum (Gautier et al. 1992). We have done such a fitting for several IRIS plates at the four wavelengths. We have selected plates with relatively bright interstellar emission (not to be affected by noise) from which we have removed the bright point sources. The effective resolution of the four IRIS bands are given in Table 1. We have checked that these values are compatible with Gaussian fitting on point sources.

\subsection{Calibration Uncertainties}

At 60 and $100 \mu \mathrm{m}$, the responsivity correction we applied at scales smaller than 1.25 depends on an interpolation between the DC/AC factor (responsivity at $5^{\prime}$ scale) and the responsivity at scale of 1.25 (deduced from the comparison of IRAS and DIRBE with scale and brightness - see $\S$ 4.3). The largest responsivity difference between these two scales is in bright regions where it reaches $\sim 20 \%$ (see Fig. 8). We estimate that the typical uncertainty on the responsivity at scales smaller than 1.25 is $\sim 5 \%$ for the four bands.

At scales larger than $1^{\circ} .25$ the IRAS data are forced to match the DIRBE data by applying an offset map (see $\S 4.5)$. The application of such an offset map takes care of responsivity variations at scales larger than $1^{\circ} .25$, but it also sets the zero level correctly. The uncertainty on the responsivity at large scales is 
4.0 10.0
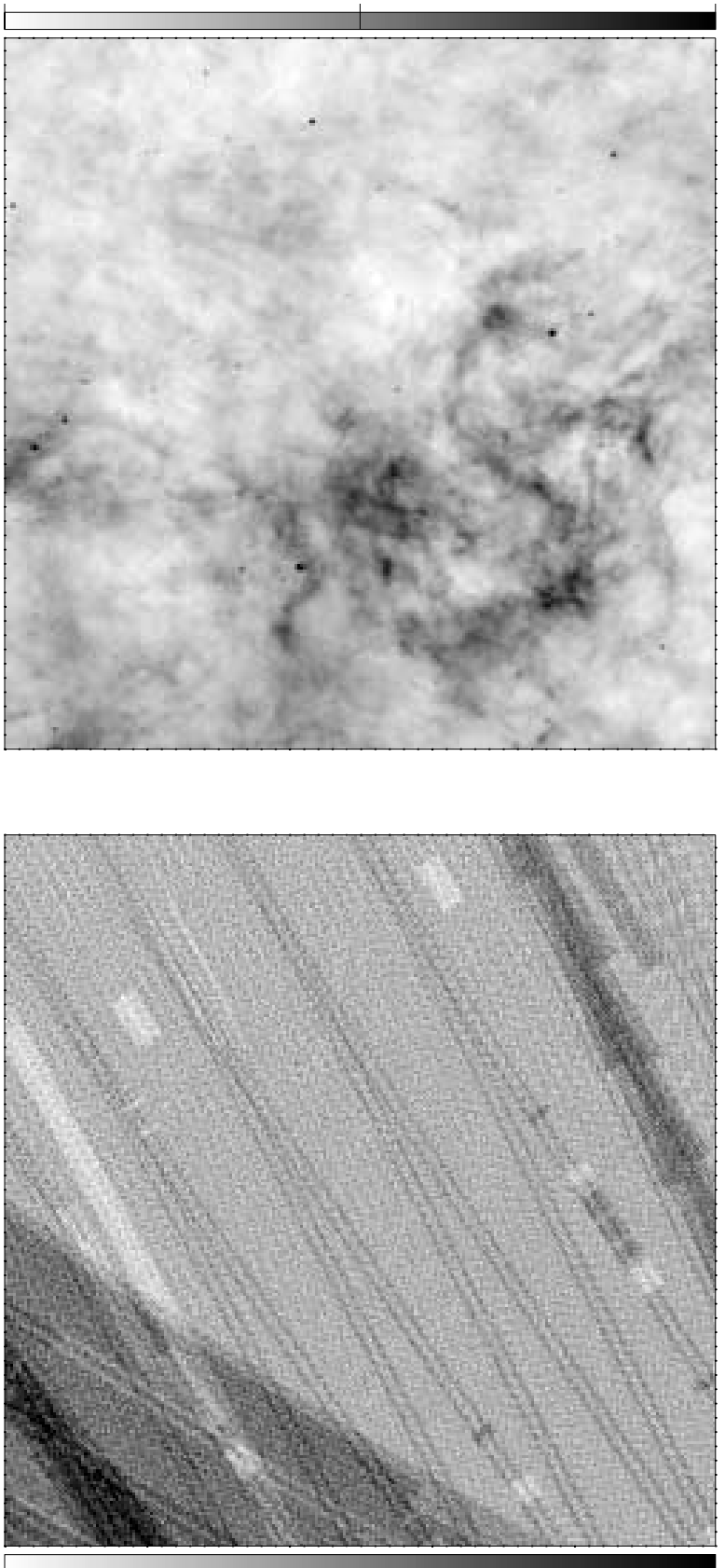

20
$16.0-0.3$

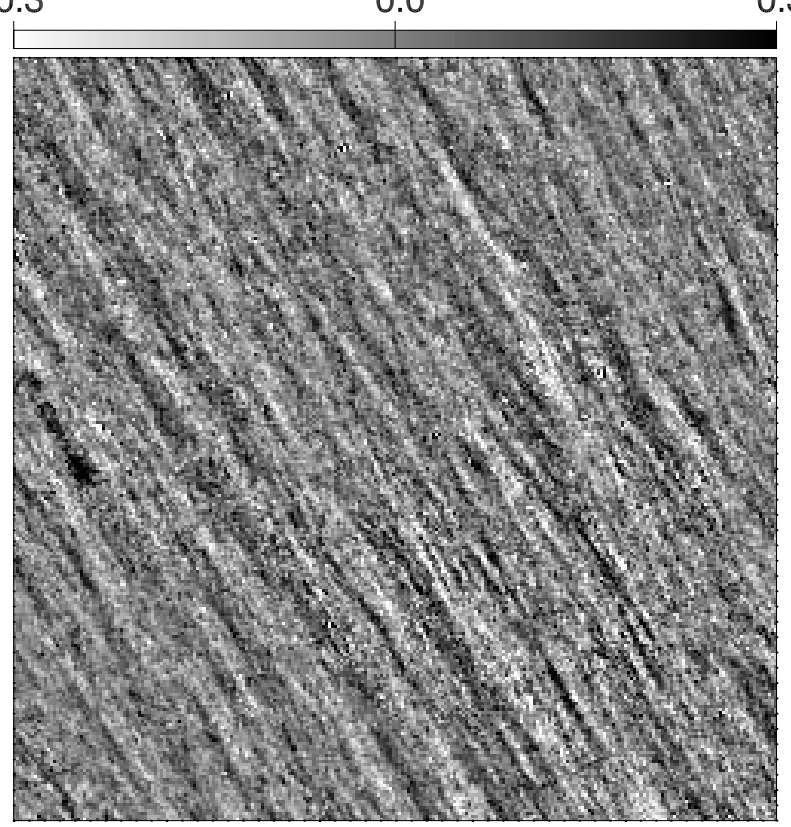


ISSA

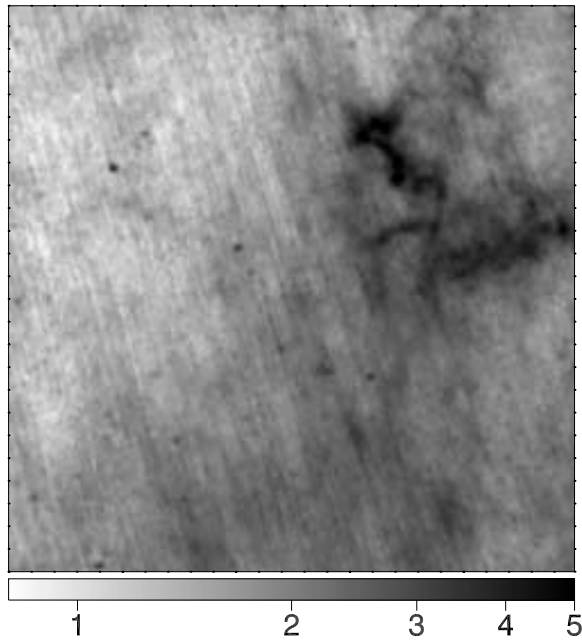

IRIS

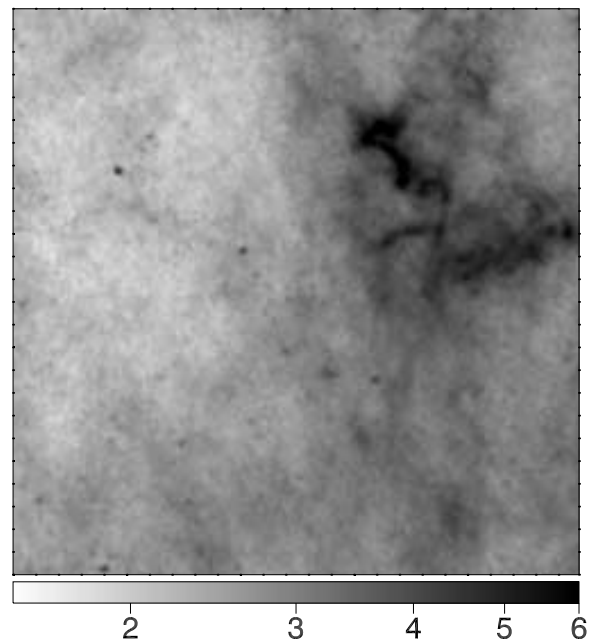

Schlegel et al.

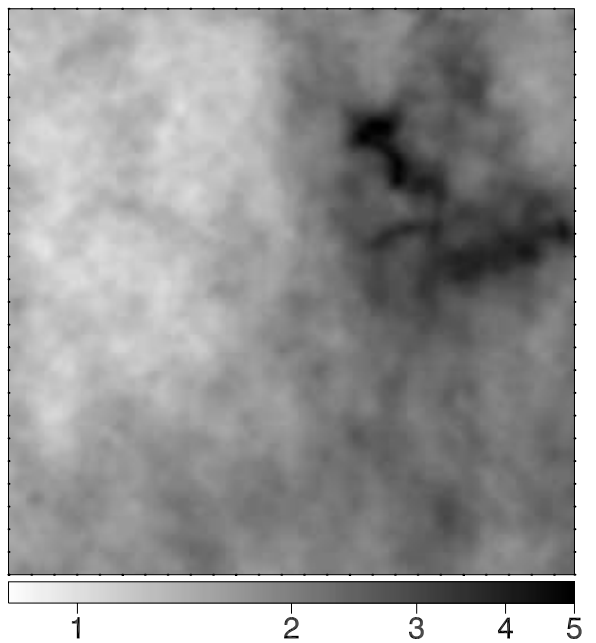

FIG. 14.-Comparison of a ISSA, IRIS, and Schlegel et al. $250 \times 250$ subimage at $100 \mu \mathrm{m}$. All the maps are in MJy sr ${ }^{-1}$. The IRIS map combined the higher angular

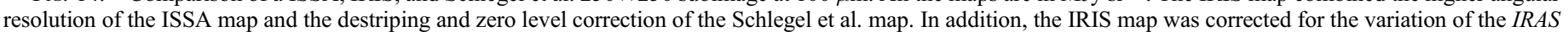

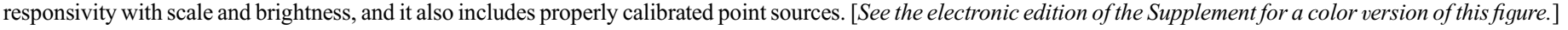

destriping and zero level of the ISSA plates in a similar fashion as we did. One of the great improvement of the IRIS $100 \mu \mathrm{m}$ data compared to Schlegel et al. (1998) is that we take into account the variation of the responsivity with brightness at scales smaller than $1^{\circ}$ (while they apply a constant factor of 0.87 ). In addition, the product given by Schlegel et al. (1998) does not include point sources.

In Figure 14 we present a comparison of a $100 \mu \mathrm{m} 250 \times$ 250 pixels subimage of the same region in ISSA, IRIS, and Schlegel et al. (1998). One striking feature of this figure is the striping that is completely removed in the IRIS and Schlegel et al. (1998) images. One feature of the IRIS product is also the fact that it includes point sources. One would also note the difference in angular resolution between the IRIS and Schlegel et al. (1998) data. Contrary to Schlegel et al. (1998) we have taken great care to keep the full ISSA resolution. The difference in angular resolution between IRIS and Schlegel et al. (1998) is well seen in Figure 15, where we compare the power spectrum of a typical $100 \mu \mathrm{m}$ map. ${ }^{5}$ There is a very good agreement

${ }^{5}$ Point sources have been subtracted from the IRIS map prior to compute the power spectrum.
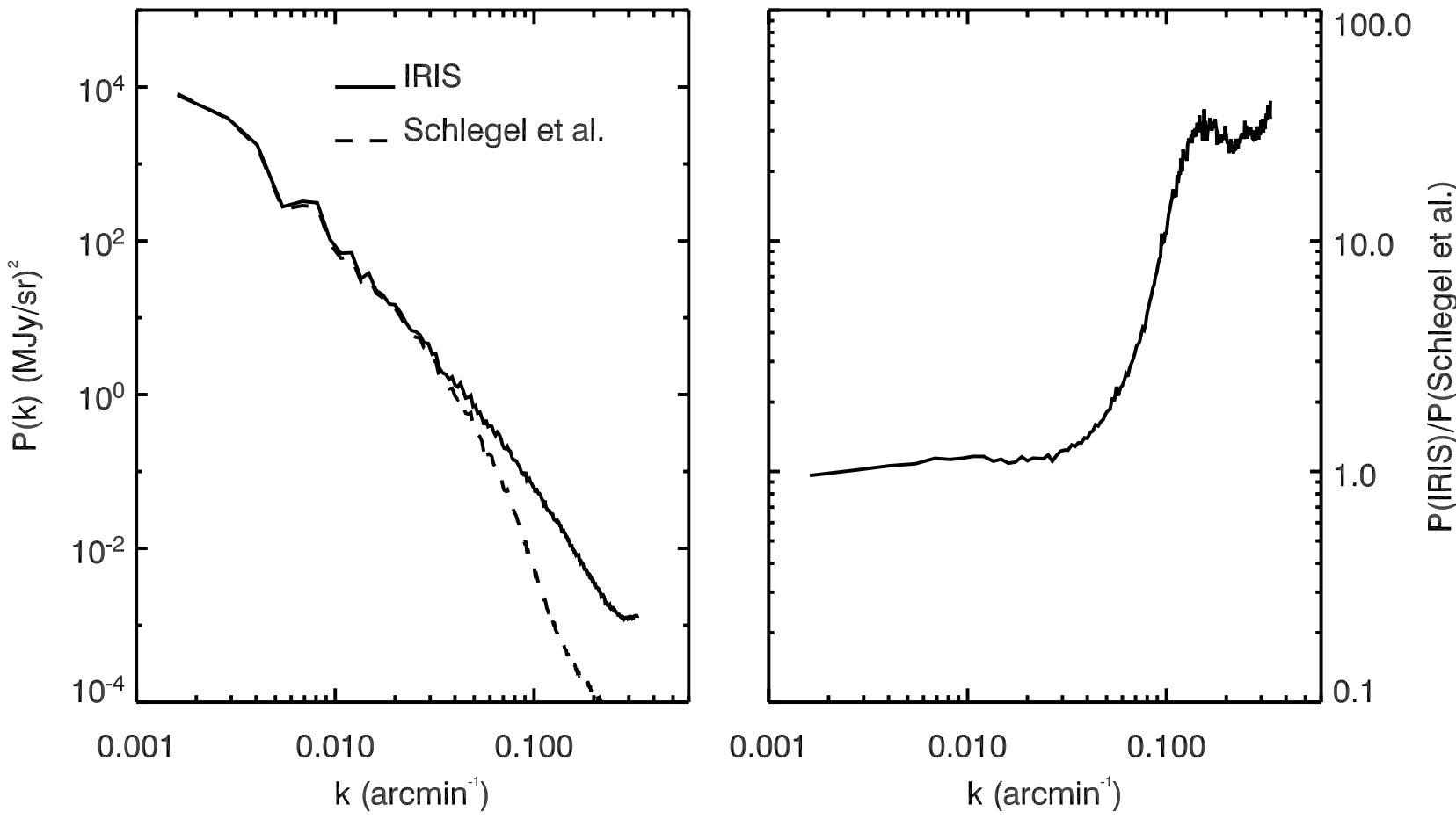

FIG. 15.-Comparison of the power spectrum of a typical $100 \mu \mathrm{m}$ image with IRIS and Schlegel et al. Left: Power spectra of the IRIS (point sources subtracted) and

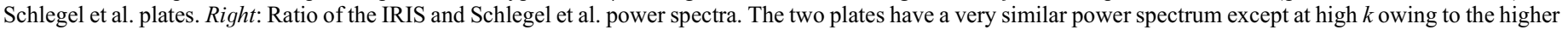
angular resolution of the IRIS plate. 
between these two data sets at scales larger than $\sim 30^{\prime}$, but there is a significant difference at smaller scales because of the difference in the responsivity correction and also to the lower resolution of the Schlegel et al. (1998) data (6.'1).

\section{CONCLUSION}

We have built a new generation of IRAS maps that are rescaled to an absolute calibration coherent with both the DIRBE and the IRAS Point Source Catalog photometry. These maps are also much less contaminated by stripes than the previous IRAS images. This new generation of IRAS maps is named IRIS for Improved Reprocessing of the IRAS Survey. The IRIS data are available online. ${ }^{6}$ The data products are detailed in Appendix $\mathrm{C}$.

Compared to the previous products (namely the ISSA and Schlegel et al. 1998), we made a major improvement of the calibration and zero level at all wavelengths (but especially at 12 and $25 \mu \mathrm{m}$ ) by comparing with DIRBE and remove significantly the stripes using an efficient iterative process that involve a wavelet decomposition of the map. Considering the relatively small uncertainty of the IRAS responsivity at subdegree scales we estimate that the overall uncertainty on the IRIS calibration

\footnotetext{
${ }^{6}$ See http://www.ias.fr/IRIS and http://www.cita.utoronto.ca/ mamd/IRIS They can also be obtained from http://www.ipac.caltech.edu and through the Centre de Données astronomiques de Strasbourg (CDS; http://www.cdsweb.ustrasbg.fr) via Aladin.
}

is dominated by the uncertainty on the DIRBE data (including the uncertainty on the zodiacal model). One other great interest of the IRIS maps is that they contain all the IRAS small-scale information (including pointlike sources) properly calibrated. With IRIS, we have reached the limit of the postprocessing of the ISSA plates. Improvements over the IRIS product will be only possible by working directly on the raw IRAS data.

The IRIS data will be of great use for the whole community. In the context of the numerous ongoing and future infrared/submm/ mm missions (Spitzer, Herschel, Planck, JWST, ASTRO-F, ...), they will be very helpful to prepare and analyze the observations. In particular, with an angular resolution comparable to the highfrequency bands of Planck, these data will be very complementary, providing unique dust templates. These data can also be used by themselves to conduct still original science. As an example, in a forthcoming paper, we will present a study of the properties of the cirrus dust emission.

We warmly thanks S. Wheelock, N. Gautier, F. Boulanger, and J.-L. Puget for the help and support they give to this work. Thanks to all the IRAS team for providing us with the great legacy ISSA product. The processing of the IRIS plates has been done on the Beowulf cluster of the Canadian Institute for Theoretical Astrophysics.

\section{APPENDIX A}

\section{POINT SOURCE IDENTIFICATION}

One important part of the postprocessing of the ISSA maps presented here is related to the identification and removal of point sources, or more generally of small-scale structures. The removal of point sources is used at several steps in the processing. As the calibration of the diffuse emission and of point sources are not the same in the ISSA plates, they have to be treated separately. Point sources identification is also used for the deglitching and destriping of the HCONs and for the determination of the zero level map.

The difficulty here is to develop a point source identification method that is equally efficient in very diffuse regions where the noise is significant and in crowded Galactic plane fields. The poor man's method to identify point sources is to apply a boxcar filtering to the image and to look for highly deviant small-scale fluctuations. For images with relatively homogeneous background the histogram of small-scale brightness fluctuation is dominated by a quasi-Gaussian core, partly due to noise, with a positive wing due to point sources (see Fig. 16). In this case point sources can easily be identified as being all the pixels with fluctuation values higher than $n \sigma$, where $\sigma$ is the width of the Gaussian core and $n$ is a given threshold.

For images with strongly varying diffuse emission this method does not work as well. In Figure 16 (middle row) we present the identification of point sources with this traditional sigma-clipping method. In this case the shape of the small-scale fluctuation histogram is very different and a much larger fraction of the pixels in this image have a small-scale fluctuations above $n \sigma$, where $\sigma$ is the width of the central component of the histogram. The result (middle-right panel) is that most of the structure in the bright parts of the image has been identified as point sources.

For bright regions the small-scale fluctuations are not dominated by noise fluctuations. The core of the small-scale fluctuation histogram comes from the diffuse emission itself. As shown by Gautier et al. (1992), the amplitude of the diffuse emission fluctuations $P(l)$ at a given scale $l$ increases with the average brightness $B_{0}$ :

$$
P(l) \propto B_{0}^{\alpha} .
$$

Gautier et al. (1992) gave $\alpha=1.5$. This indicates clearly that the fluctuations level of the diffuse emission increases with brightness. This is clearly seen in Figure 16, where bright regions have generally stronger small-scale fluctuations.

To take this effect into account we rather use the contrast of the small-scale fluctuations (i.e., fluctuations/brightness) to identify point sources, instead of the small-scale fluctuation level itself. This way we take into account the variation of the diffuse emission fluctuations with brightness (see eq. [A1]). The result of this method is shown on the bottom row of Figure 16; much less pixels were clipped. This method is much more flexible than the traditional sigma-clipping method as it adapts itself to the background level.

At this point it is important to mention that the contrast method cannot be applied in diffuse regions, where noise contributes significantly to the small-scale brightness fluctuations. For each band we used the traditional sigma-clipping for regions with boxcar 

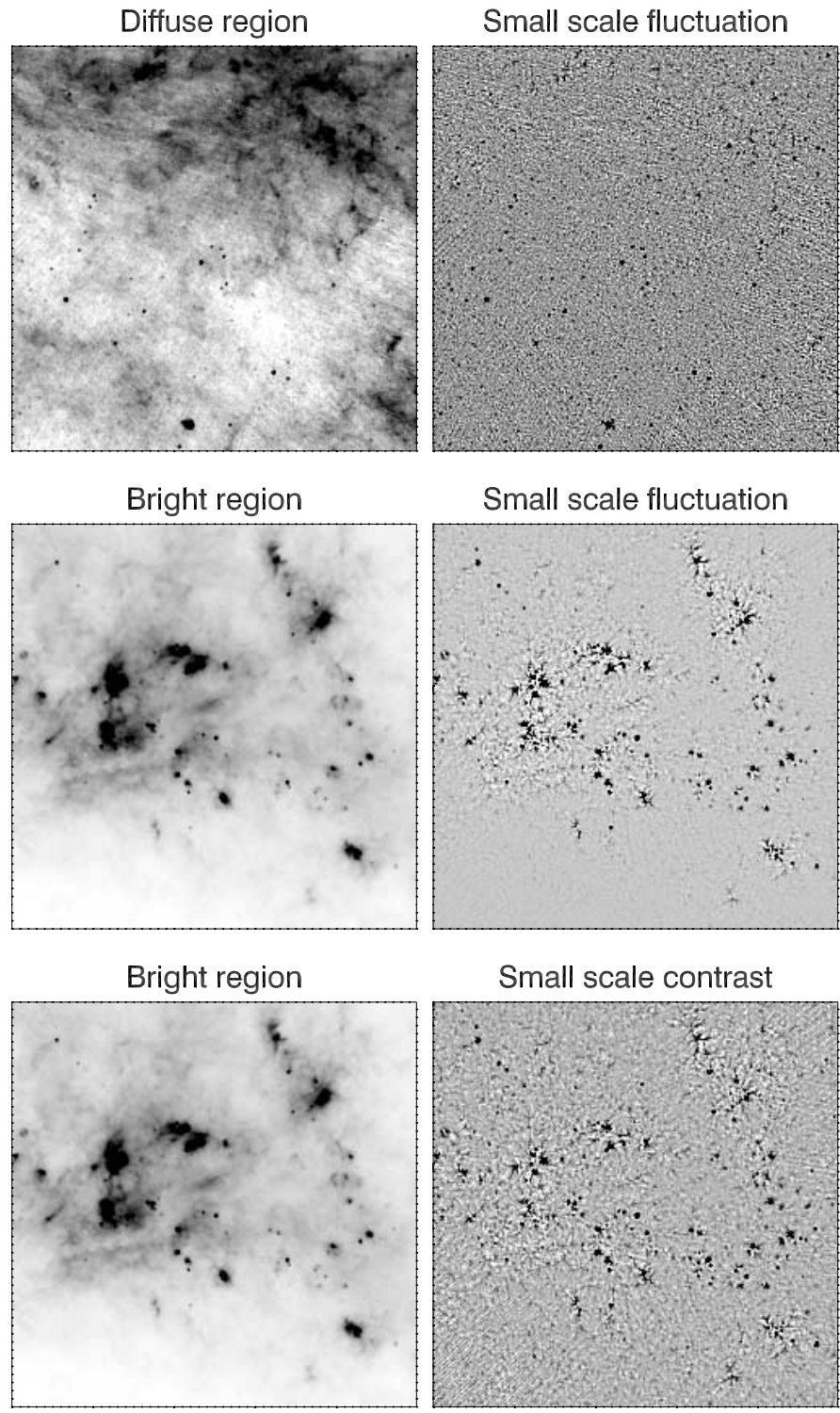
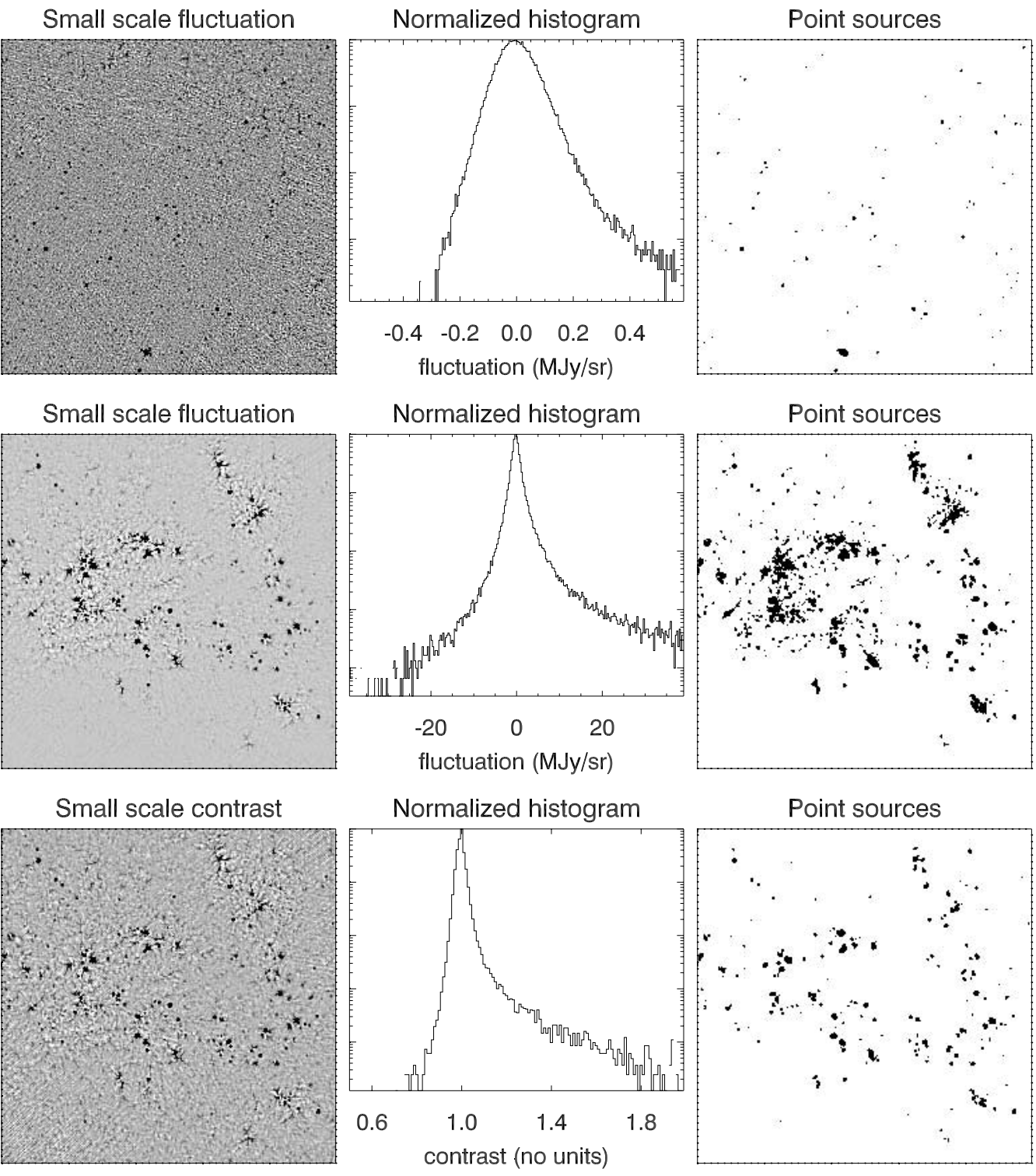

FIG. 16.-Example of point source identification. 

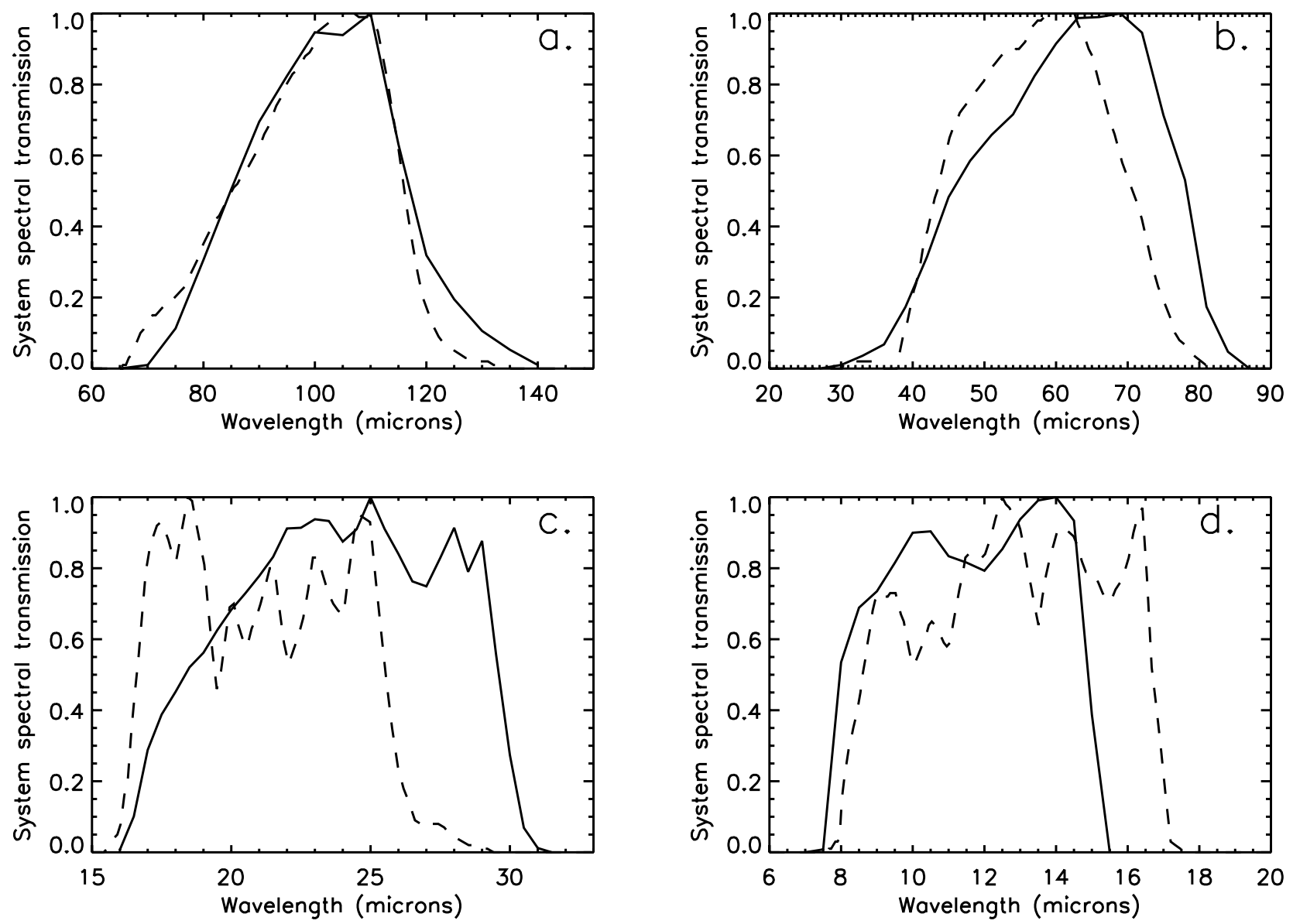

FIG. 17.-System spectral responses for the IRAS (solid lines) and DIRBE (dashed lines) 100, 60, 25, and $12 \mu \mathrm{m}$ bands ( $a-d$, respectively).

filtered brightness lower than 3, 3, 4, and $4 \mathrm{MJy} \mathrm{sr}^{-1}$ at 12, 25, 60, and $100 \mu \mathrm{m}$, respectively. For regions with brightness higher than those values we used the contrast method to identify point sources.

\section{APPENDIX B}

\section{IRAS AND DIRBE FILTERS}

Following the IRAS convention, the DIRBE spectral intensities $I_{\nu}$ are expressed in $\mathrm{MJy} \mathrm{sr}^{-1}$ at fixed nominal frequencies assuming the source spectrum is $\nu I_{\nu}=$ constant (i.e., constant intensity per logarithmic frequency interval).

Since the source spectrum has not a constant intensity per logarithmic frequency interval, a color correction has to be applied to obtain an accurate intensity. The color correction factor is defined such that

$$
I_{\nu_{0}}(\text { actual })=I_{\nu_{0}}(\text { quoted }) / \mathrm{cc}
$$

where $I_{\nu_{0}}$ (actual) is the actual specific intensity of the sky at frequency $\nu_{0}, I_{\nu_{0}}$ (quoted) is the corresponding value given with the IRAS or DIRBE convention and $\nu_{0}$ is the frequency corresponding to the nominal wavelength of the considered band. With these definitions,

$$
\mathrm{cc}=\frac{\int\left(I_{\nu} / I_{\nu_{0}}\right)_{\mathrm{actual}} R_{\nu} d \nu}{\int\left(\nu_{0} / \nu\right) R_{\nu} d \nu}
$$

where $\left(I_{\nu} / I_{\nu_{0}}\right)_{\text {actual }}$ is the actual specific intensity of the sky normalized to the intensity at frequency $\nu_{0}$ and $R_{\nu}$ is the system spectral response. The spectral responses are given in Figure 17 for the four IRAS and DIRBE bands.

When we compare the DIRBE and IRAS intensities, we compare $I_{\nu_{0}}$ (quoted) $)_{\operatorname{DIRBE}}$ and $I_{\nu_{0}}(\text { quoted })_{I R A S}$. For the same point on the sky and in the same beam (i.e., when IRAS data are convolved by the DIRBE beam), $I_{\nu_{0}}(\text { actual })_{\text {DIRBE }}=I_{\nu_{0}}(\text { actual })_{I R A S}$. This leads to

$$
\frac{I_{\nu_{0}}(\text { quoted })_{I R A S}}{I_{\nu_{0}}(\text { quoted })_{\text {DIRBE }}}=\frac{\mathrm{cc}_{I R A S}}{\mathrm{cc}_{\text {DIRBE }}} .
$$


TABLE 3

Ratio of IRAS to DiRbE Color Corrections for Spectra IN THE FORM $I_{\nu} \propto \nu^{\beta} B_{\nu}(T)$

\begin{tabular}{|c|c|c|}
\hline $\begin{array}{l}\text { Wavelength } \\
\qquad(\mu \mathrm{m})\end{array}$ & $(T[\mathrm{~K}], \beta)$ & $\mathrm{cc}_{I R A S} / \mathrm{cc}_{\text {DIRBE }}$ \\
\hline \multirow[t]{9}{*}{100} & $(14,2)$ & 1.1019 \\
\hline & $(16,2)$ & 1.0737 \\
\hline & $(18,2)$ & 1.0525 \\
\hline & $(20,2)$ & 1.0354 \\
\hline & $(22,2)$ & 1.0211 \\
\hline & $(14,1.5)$ & 1.1146 \\
\hline & $(16,1.5)$ & 1.0852 \\
\hline & $(22,1.5)$ & 1.0321 \\
\hline & $(-,-)$ & 1.0510 \\
\hline \multirow[t]{5}{*}{60} & $(40,1)$ & 1.0476 \\
\hline & $(60,1)$ & 0.9452 \\
\hline & $(40,0)$ & 1.1187 \\
\hline & $(60,0)$ & 0.9947 \\
\hline & $(-,-)$ & 1.1045 \\
\hline \multirow{4}{*}{ 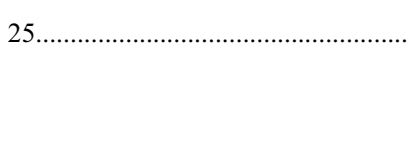 } & $(-, 0)$ & 0.8941 \\
\hline & $(-, 1)$ & 0.8036 \\
\hline & $(-, 2)$ & 0.7265 \\
\hline & $(-,-)$ & 0.9728 \\
\hline \multirow[t]{4}{*}{12} & $(-, 0)$ & 1.0868 \\
\hline & $(-, 1)$ & 1.1723 \\
\hline & $(-, 2)$ & 1.2552 \\
\hline & $(-,-)$ & 1.0938 \\
\hline
\end{tabular}

Notes.-When no values are given for the temperature, the considered spectrum is in the form $I_{\nu} \propto \nu^{\beta}$. When no values are given for both the temperature and spectral index, the considered spectrum is the standard diffuse ISM spectrum for $10^{20}$ atoms $\mathrm{cm}^{-2}$.

Therefore, when comparing the DIRBE and IRAS data to calibrate the IRAS data, we should correct the DIRBE data by the ratio of color corrections. This ratio is varying across the sky. It is quite easy to compute it in the far-infrared at large scales, where we can infer the dust emissivity and temperature using DIRBE data (using an iterative procedure). At smaller scales and at 12 and $25 \mu \mathrm{m}$, it is impossible to compute this ratio since we do not know the exact shape of the intrinsic energy distribution for most of the sky. As an example, ratios of color corrections are given for different spectral energy distribution in Table 3.

\section{APPENDIX C}

\section{DATA PRODUCTS}

The "standard" IRIS data ("standard" means for the processing described in this paper) are available online (see footnote 1). On the two sites mentioned in the footnote the data are available in the original ISSA format: 430 maps of $500 \times 500$ pixels for each band. The individual HCON can also be downloaded from these sites. In the near future we expect to provide Healpix version of these maps on these two sites. We also expect to provide versions of the maps without point sources and with an improved zodiacal light subtraction.

The IRIS data product is also available through the Centre de Données astronomiques de Strasbourg (CDS) and at the Infrared Processing and Analysis Center (IPAC) (see footnote 6).

Bennett, C. L., et al. 2003, ApJS, 148, 97

Bernard, J. P., et al. 1999, A\&A, 347, 640

Boulanger, F., Falgarone, E., Puget, J. L., \& Helou, G. 1990, ApJ, 364, 136

Boulanger, F., \& Pérault, M. 1988, ApJ, 330, 964

Coulais, A., \& Abergel, A. 2000, A\&AS, 141, 533

Draine, B. T., \& Lazarian, A. 1998, ApJ, 494, L19

Finkbeiner, D. P., Davis, M., \& Schlegel, D. J. 1999, ApJ, 524, 867

Fouks, B. I., \& Schubert, J. 1995, Proc. SPIE, 2475, 487

Gautier, T. N. I., Boulanger, F., Perault, M., \& Puget, J. L. 1992, AJ, 103, 1313

Hauser, M. G., et al. 1998, ApJ, 508, 25

Kelsall, T., et al. 1998, ApJ, 508, 44

Lagache, G. 2003, A\&A, 405, 813

Lagache, G., Abergel, A., Boulanger, F., \& Puget, J. L. 1998, A\&A, 333, 709

\section{REFERENCES}

Low, F. J., et al. 1984, ApJ, 278, L19

Miville-Deschênes, M. A., Boulanger, F., Joncas, G., \& Falgarone, E. 2002a, A\&A, 381, 209

Miville-Deschênes, M. A., Lagache, G., \& Puget, J. L. 2002b, A\&A, 393, 749

Miville-Deschênes, M. A., Levrier, F., \& Falgarone, E. 2003, ApJ, 593, 831

Neugebauer, G., et al. 1984, ApJ, 278, L1

Schlegel, D. J., Finkbeiner, D. P., \& Davis, M. 1998, ApJ, 500, 525

Starck, J. L., \& Murtagh, F. 1998, PASP, 110, 193

Stepnik, B., et al. 2003, A\&A, 398, 551

Vinokurov, L. A., \& Fouks, B. I. 1991, Soviet Phys. Semicond., 25, 1207

Wheelock, S., et al. 1993, ISSA Explanatory Supplement, Technical Report (Pasadena: IPAC) 